\title{
Direct effects of boundary permeability on turbulent flows: observations from an experimental study using zero-mean-shear turbulence
}

\author{
Mark W. McCorquodale $\nmid$ and R. J. Munro $\ddagger$ \\ Faculty of Engineering, The University of Nottingham, Nottingham, NG7 2RD, UK
}

(Received xx; revised $\mathrm{xx}$; accepted $\mathrm{xx}$ )

The interaction of zero-mean-shear turbulence (generated using an oscillating grid) with solid and permeable boundaries is studied experimentally. The influence of wall permeability is characterised using the permeability Reynolds number, $R e_{K}$, which represents the ratio of the typical pore size in the permeable media to a viscous length scale. Instantaneous velocity measurements, obtained using two-dimensional particle imaging velocimetry, are used to study the effect boundary permeability has on the rms of fluctuating velocity components, the vertical flux of turbulent kinetic energy (TKE) and conditional turbulent statistics associated with events in which intercomponent energy transfer is concentrated. When $R e_{K} \lesssim 0.2$ the boundary acts as if it were impermeable; results indicate the interaction is dominated by the kinematic blocking effect of the boundary on the boundary-normal TKE flux, with additional mechanisms acting through intercomponent energy transfer. The results show these mechanisms are inhibited as $R e_{K}$ increases, due to the transportation of turbulent energy into the porous media as the macroscopic blocking condition is relaxed, thereby reducing TKE within the boundaryaffected region and inhibiting the formation of high-pressure stagnation events that are responsible for intercomponent energy transfer. The results illustrate how the turbulence structure above a permeable boundary is sensitive to the blocking effect on the boundarynormal turbulent velocity. In light of these results, we propose that further analysis is required to establish the validity of a commonly-used model of the boundary conditions enforced at the boundary of porous media, in which a no-penetration boundary condition on the boundary-normal velocity component is proposed.

\section{Introduction}

Turbulence interacting with a boundary is a fundamental topic of interest for scientists working within a broad range of fields. [Here we use the term 'boundary' to refer to the plane separating an impermeable surface, or the surface of a porous media, and an adjacent layer of fluid]. As a canonical case, much research has been devoted to understanding the interaction of a turbulent flow with a flat (smooth or rough) impermeable surface aligned with the plane of the boundary parallel to the mean velocity of the flow, which we refer to as turbulent channel flow. This research has yielded significant developments in our understanding of the structure of the turbulent boundary

$\dagger$ Email address for correspondence: mark.mccorquodale2@nottingham.ac.uk ¥ Email address for correspondence: rick.munro@nottingham.ac.uk 
layer (TBL) and the mechanisms by which turbulence is produced within the TBL. However, many natural and engineering materials are permeable, and thus the structure of the TBL adjacent to the surface of a porous media is also of great interest. In this case, the canonical problem consists of turbulent channel flow in which a permeable media is bounded on (at least) one side by a turbulent flow. In such situations a mean stream-wise flow exists both above the surface of the porous media and also within the porous media itself. Recent studies have significantly improved our understanding of this flow and reveal the breadth of related problems, with applications as diverse as monitoring and improving water quality in streams and coastal regions (Manes et al. 2009; Voermans et al. 2017); improving the efficiency of engineering devices used for heat and mass transfer, such as catalytic converters and heat exchangers (Kuwata \& Suga 2017); and the design of novel surfaces for drag reduction purposes (Rosti et al. 2018).

In this canonical problem simple well-established models (such as Darcy's law or Brinkman 1947) can be used to describe the bulk flow (i.e. volume averaged flow) within the permeable medium. The theoretical basis for these well-established models has been extensively documented in the works of, amongst others, Whitaker, Nield, Bear and Gray (see, for example, Whitaker 1999; Nield \& Bejan 2013; Bear 2013; Hassanizadeh \& Grey 1979, 1990). However, more complex models are required to describe the flow close to the edge of the permeable media where there exists an "interface region", above and below the surface of the permeable medium, in which the flow characteristics depend on both the flow in the permeable medium and in the adjoining unconfined flow (see, for example, Bottaro 2019). Models to describe flow in the interface region have been proposed through use of a boundary condition that describes fluid velocities in the direction of bulk flow - the so called "slip velocity" (see, for example, Beavers \& Joseph 1967; Hanh et al. 2002). The slip velocity represents an empirical approximation of the boundary conditions imposed by the surface of a permeable medium on the bulk flow. That is, at the surface of a permeable media the no-slip and no-penetration boundary conditions are enforced along the convoluted surface of the elements that constitute the permeable medium but are not enforced within the voids of the permeable medium, resulting in a macroscopic relaxation of the boundary conditions. Such conditions are challenging to model since it is typically infeasible to directly resolve the pore-scale structure of a permeable medium in studies using computational fluid dynamics (Rosti et al. 2015).

Evidently the flow in the interface region is influenced by the permeability of the porous medium - this effect is characterised using the permeability Reynolds number, defined as $R e_{K} \equiv u_{*} \sqrt{K} / \nu$, where $u_{*}$ and $K$ denote the friction velocity and the absolute permeability $\dagger$ respectively. This approach was initially introduced by Hanh et al. (2002) and subsequently refined by Breugem et al. (2006) in the context of turbulent channel flows. Results from these studies indicate that when $R e_{K} \ll 1$ a surface is effectively impermeable, with flow characteristics similar to that at an impermeable surface, as viscous sublayers over separate elements of the porous medium are thick enough to coalesce and form a continuous viscous sublayer covering the horizontal plane separating the porous media and fluid above (Breugem et al. 2006). When $R e_{K} \gg 1$ a surface is highly permeable, in which viscous effects are of minor importance (Breugem et al. 2006)

$\dagger$ The absolute (or intrinsic) permeability is a parameter characterising the permeability of a porous media that is independent of fluid properties (see, for example, Lage 1998). For homogeneous isotropic porous media, the absolute permeability $K$ is a scalar quantity that can be related to the hydraulic conductivity $k_{p}$, which characterises the ease with which a given fluid can travel through a permeable media, through the expression $K=\mu k_{p} /(\rho g)$, where $\mu, \rho$ and $g$ denote dynamic viscosity, fluid density and gravitational acceleration respectively (see, for example, Bear 2013, p. 132). 
such that turbulent eddies may be able to penetrate the permeable boundary (Manes et al. 2009). Therefore, depending upon the flow conditions, behaviour at a permeable surface can be thought of as intermediate between the limits of a solid surface and unconfined flow.

Recent channel flow studies (for example Breugem et al. 2006; Suga et al. 2010; Kuwata \& Suga 2017; Rosti et al. 2015; Voermans et al. 2017; Kim et al. 2020) are beginning to reveal a more complete picture of the changes in boundary layer structure that occur within the interface region. The relaxation of the no-slip and no-penetration boundary conditions on the horizontal plane separating the surface of the porous layer and the fluid above may give rise to the development of Reynolds shear stress close to the surface of the porous media. This observation is associated with an increase in the wall-normal Reynolds stress despite there being a reduction in the peak of streamwise Reynolds stress. The development of the Reynolds shear stress close the boundary is of particular importance as it gives rise to an increase in surface shear stress or skin friction of the boundary (Breugem et al. 2006; Manes et al. 2009; Yokojima 2011; Kuwata \& Suga 2016).

The development of Reynolds shear stress close to the boundary is thought to be related to the presence of vortical structures that originate from Kelvin-Helmholtz type instabilities (Breugem et al. 2006; Manes et al. 2011; Kuwata \& Suga 2017; Efstathiou \& Luhar 2018; Rosti et al. 2018). This instability arises as a result of the development of an inflection in the mean velocity profile close to the boundary. The presence of these vortical structures is part of a wider change in structure and dynamics in the boundary layer; quasi-streamwise vortices (such as hairpin vorticies) and high- and lowspeed streaks typically observed in a TBL above an impermeable surface have been reported to be weakened, or to not form at all, above a permeable boundary (Hanh et al. 2002; Breugem et al. 2006; Yokojima 2011; Suga et al. 2011, 2017; Rosti et al. 2015). As a means of explanation, it has been noted that a strong mean velocity gradient (strong mean-shear) is required for the existence of the high- and low-speed streak structure and this condition is not satisfied above highly permeable boundaries due to the relaxation of the no-slip condition (Breugem et al. 2006). In addition, because of the weakening of the wall-blocking effect, strong wall-normal velocities are present near the permeable surface and this also prevents the development of elongated streaky structures (Breugem et al. 2006). Furthermore, it has been proposed that strong wallward motions (sweeps) are able to penetrate a porous media (Pokrajac \& Manes 2009), within which their kinetic energy is dissipated such that the corresponding ejections (to balance the mass flux) are emitted with reduced kinetic energy (Suga et al. 2011). The intensity of these upwelling and downwelling events is further influenced by the passage of large-scale motions in the unconfined flow above the permeable media. Energetic downflow events (sweeps) are associated with large-scale regions of high streamwise momentum in the unconfined flow, whilst upflow events (ejections) are associated with large-scale regions of low streamwise momentum in the unconfined flow (Kim et al. 2020).

Measurements of terms of the transport equations of TKE and the Reynolds stress tensor provide further insight and indicate that the transport of TKE towards the boundary is a dominant effect close to the boundary (see for example Breugem et al. 2006; Yokojima 2011; Kuwata \& Suga 2016). In particular, transport by pressure fluctuations allows TKE to be transported much deeper within a permeable medium than by turbulent transport, which is limited to a thin interface region of the porous medium (Breugem et al. 2006; Manes et al. 2009; Kuwata \& Suga 2016). The increased transport by pressure fluctuations has been attributed to the intensification of pressure fluctuations by the Kelvin-Helmholtz instability and the weakening of the wall-blocking effect (Breugem et al. 2006; Kuwata \& Suga 2016). The intensification of pressure fluctuations also gives 
rise to an increase in the intercomponent energy transfer that occurs close to the surface of the porous media (Breugem et al. 2006; Kuwata \& Suga 2016).

In summary, in a turbulent channel flow, the differences between the structure of the TBL adjacent to the surface of a porous media and an impermeable media are principally attributed to two physical processes: (i) penetration of turbulent eddies into the porous media as a result of a relaxation of the macroscopic no-slip and no-penetration boundary conditions; (ii) a reduction in mean-shear close to the boundary due to flow occurring within the permeable medium itself, which gives rise to an inflection in the mean velocity profile and an associated Kelvin-Helmholtz type instability. Thus, aspects of the dynamics governing the structure of the flow that forms in a permeable channel are relatively well understood.

However, results obtained through studying this canonical problem do not provide physical insight into the dynamics governing the interaction of the surface of a porous media with a wide range of other turbulent flows. That is, in a permeable channel flow the influence of the porous media acts on turbulence both (i) directly, through the action of the no-slip and no-penetration boundary conditions (on the surfaces of the solid elements that comprise the porous media) on turbulent fluctuations, and (ii) indirectly, through the production of TKE by maintaining mean velocity gradients in the interface region. Consequently, it is very difficult to use the results obtained from this canonical problem to develop a general framework for understanding the interaction of turbulent flows with porous media since, in these flows, it is impossible to distinguish the direct effects of boundary permeability on turbulent fluctuations from the indirect effects described above. This limitation necessitates the study of a much broader range of turbulent flows interacting with porous media, in order to develop a more comprehensive understanding of this phenomenon.

Of course, there already exists a body of literature investigating the interaction of different types of turbulent flows with the surface of a porous media; examples include permeable pipes (Wagner \& Friedrich 1998, 2000) and the impact of a jet on a surface or screen (Cant et al. 2002; Webb \& Castro 2006; Musta \& Krueger 2015). However, these studies still suffer from the same fundamental limitation as the permeable channel flow; in these flows it is impossible to distinguish the direct effects of boundary permeability on the turbulent fluctuations from the indirect effects associated with TKE production and therefore it is difficult to apply the results in contexts different from the specific flow considered. Instead, what is needed is a means of separating the direct and indirect effects of a porous surface on turbulent fluctuations; we believe a detailed understanding of the direct effects of boundary permeability on turbulent fluctuations is necessary to fully understand the dynamics governing a wide-range of turbulent flows interacting with porous media.

One can isolate the direct effects of the surface of a porous media on turbulent fluctuations by studying a turbulent flow in which there is negligible mean shear at the surface. That is, in a "zero-mean-shear" turbulent flow the presence of the permeable surface acts directly on the turbulent fluctuations through the action of the no-slip and no-penetration boundary conditions, but does not generate indirect effects on the turbulent fluctuations such as the production of TKE. It should also be noted that, in addition to their use in deriving new insight into physical processes, zero-mean-shear flows also serve as realistic idealisations of some engineered flows. For example, flows that exhibit large turbulent fluctuations, with only small mean flow velocities, interacting with porous media can be found in cleaning and decontamination processes (Connolly et al. 1983; Valsaraj et al. 1997; Orlins \& Gulliver 2003; Masaló et al. 2008).

Currently, the effects of permeability in the interaction of a zero-mean-shear turbulent 
flow with a surface are unknown; studies of the interaction of zero-mean-shear turbulence with a surface in which the applied boundary conditions are consistent with a real permeable boundary are, as far as we are aware, unprecedented. In the most closely related available study, the effects of a "perfectly permeable boundary" on initially homogeneous isotropic zero-mean-shear turbulence were analysed using Direct Numerical Simulation (DNS) (Perot \& Moin 1995). Note that at a "perfectly permeable boundary" the no-slip condition is enforced at the boundary but the no-penetration condition is not enforced. Perot \& Moin (1995) found that both boundary-tangential and boundarynormal Reynolds stresses were monotonically reduced by the boundary despite the absence of a blocking condition on the boundary-normal velocity component. The reduction in boundary-normal Reynolds stress was reported to be a result of intercomponent energy transfer from the boundary-normal Reynolds stress to the boundary-tangential Reynolds stresses, which Perot \& Moin (1995) attributed to a viscous dissipative mechanism (i.e. as at an impermeable boundary). The energy lost close to the boundary from the boundarynormal Reynolds stress (through the pressure-strain term) was reported to be replenished with turbulent energy from regions further from the wall by turbulent transport and pressure transport (Perot \& Moin 1995). Aspects of these results exhibit similarities to studies investigating permeable channel flows, however the nature of the applied boundary conditions render the results of Perot \& Moin (1995) hard to interpret for a natural permeable material.

In this study, we report results from experiments using oscillating-grid turbulence to explore the interaction of turbulence with both solid and permeable boundaries under conditions in which the flow is dominated by turbulent fluctuations, with only small mean flow velocities, thereby closely approximating zero-mean-shear conditions at the boundaries. In $\S 2$ we describe the experimental set-up and define a permeability Reynolds number $R e_{K}$ suitable for use in zero-mean-shear turbulence. In $\S 3$ we present experimental results that describe how the turbulent velocity components are affected by the solid and permeable boundaries, including measurements of the r.m.s velocity components, vertical flux of TKE and mean dynamic pressure gradient, which provide evidence of the mechanisms governing the interaction. In $\S 4$ we present results of a statistical analysis of blocked eddy motions in the interface region, which provide further evidence of the governing mechanisms. The interpretation of these results in the context of models used to describe flow in the interface region is discussed in $§ 5$. Conclusions are made in $\S 6$.

\section{Experiments}

\subsection{Apparatus}

A schematic view of the experimental set-up is shown in figure 1 . The experiments were conducted in a transparent acrylic box with internal dimensions $35.2 \mathrm{~cm} \times 35.2 \mathrm{~cm} \times 48 \mathrm{~cm}$ (henceforth denoted the 'outer box', see figure 1a), which was filled with a salt-water solution of uniform density $\rho=1.028 \mathrm{~g} / \mathrm{cm}^{3}$. A grid made of stainless steel, consisting of an array of $7 \times 7$ bars with square cross-section of $1 \mathrm{~cm}$ width and mesh spacing $M=5 \mathrm{~cm}$ (i.e. solidity $36.4 \%$ ), was suspended inside the outer box with its plane horizontal. The edge conditions for the grid were chosen such that the tank walls were planes of symmetry, as shown in figure 1(b). The grid was attached to the base of a stainless steel drive shaft (of $1 \mathrm{~cm}$ diameter) and was oscillated vertically with constant frequency $f$ and stroke $S$ (see figure 1a). Here the stoke $S$ is defined as equal to the amplitude of the grid's motion. An open-ended inner box, constructed from $0.5 \mathrm{~cm}$ thick transparent acrylic, with internal 


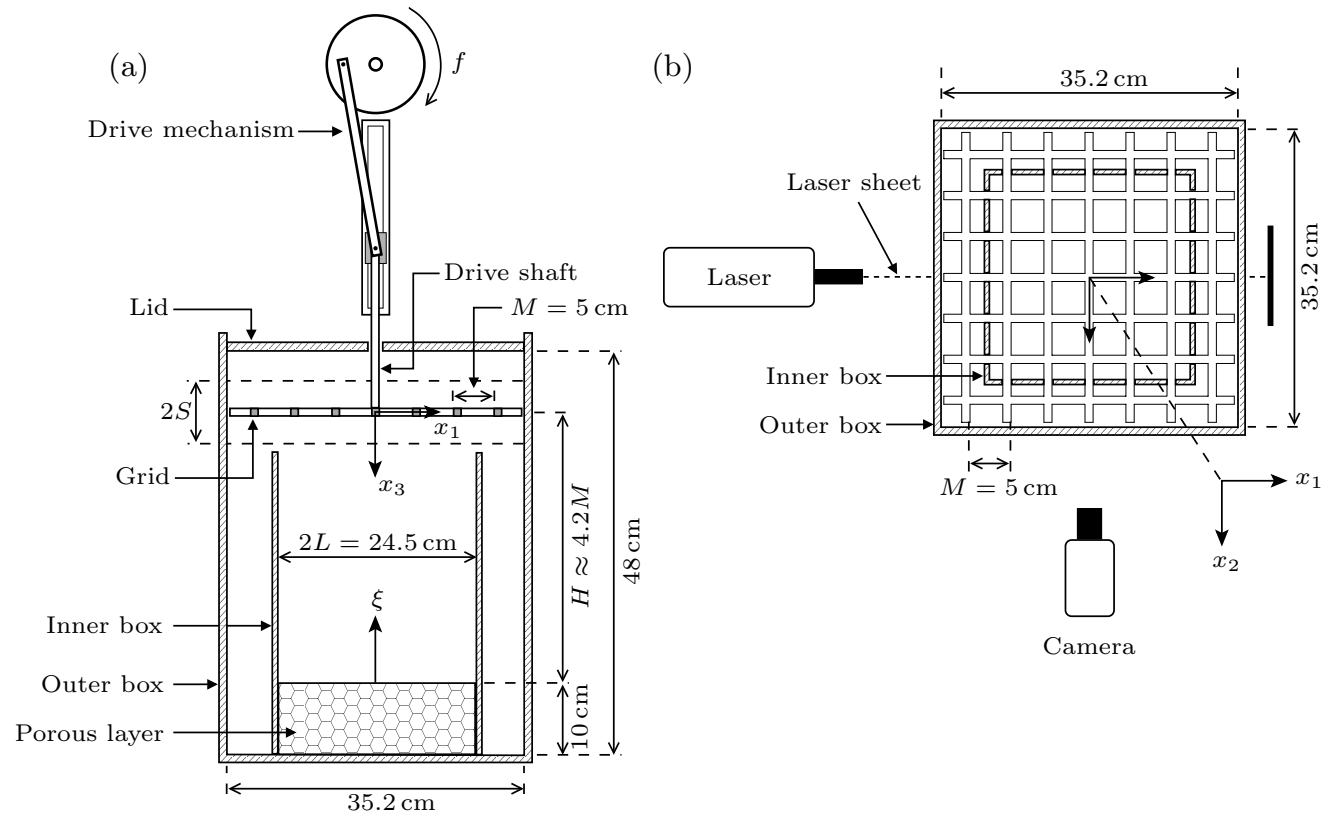

(c)

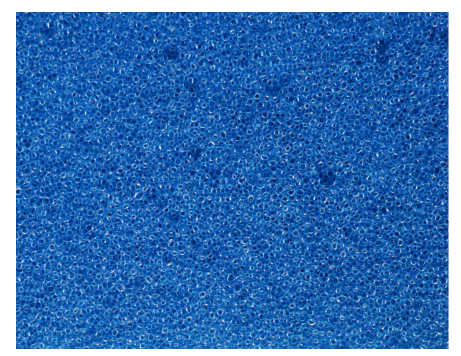

(d)

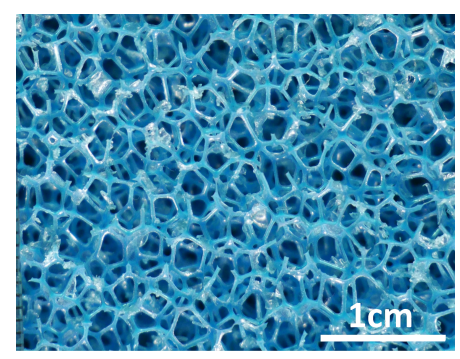

FiguRE 1. (a,b) Sketches showing the key components of the experimental set-up, including the positioning of the horizontal grid, the porous layer and the inner and outer boxes; (a) shows a sides view and $(\mathrm{b})$ shows a plan view. Also shown are the coordinate directions $\left(x_{1}, x_{2}, x_{3}\right)$, and the vertical distance from the permeable boundary, denoted $\xi=H-x_{3}$. The permeable media used are shown in $(\mathrm{c}, \mathrm{d}) ; 60$ pores per inch (PPI) and 10PPI foams are shown, respectively. Each permeable media is shown at the same scale (a reference scale is provided in (d) which applies to both images).

dimensions $24.5 \mathrm{~cm} \times 24.5 \mathrm{~cm} \times 26.5 \mathrm{~cm}$, was fixed centrally on-plan at the base of the tank. We will henceforth let $2 L=24.5 \mathrm{~cm}$ denote the internal width of the inner box. The grid was positioned so that when at the bottom of its stroke it was $1 \mathrm{~cm}$ above the top of the inner box. The vertical walls of the inner box were located equidistant between the outermost and second-outermost bars of the grid, as shown in figure 1(b). The use of an inner box of this design has been shown to systematically reduce the mean flow present within the turbulence produced (McCorquodale \& Munro 2018b).

Two different porous media were used for this study, in addition to a solid impermeable surface. The impermeable surface was formed by inserting a solid acrylic plate into the inner box at a depth $H \approx 4.2 M$ below the grid's mean position (see figure 1a). A tight fit was ensured between the plate and the inner box by use of thin neoprene seals, set into the perimeter of the plate. The porous media were comprised of $2.5 \mathrm{~cm}$ thick sheets of 


\begin{tabular}{ccccc} 
Media used & $k_{p}(\mathrm{~m} / \mathrm{s})$ & $K\left(\mathrm{~m}^{2}\right) \times 10^{-8}$ & $l_{\text {cell }}(\mathrm{mm})$ & $\operatorname{Re}_{K}$ \\
\hline Impermeable plate & 0 & 0 & 0 & 0 \\
60PPI foam & $0.0997(0.0951,0.107)$ & $1.07(1.02,1.15)$ & $0.62-0.86$ & $0.04,0.06,0.08,0.09,0.10$ \\
10PPI foam & $1.6(1.3,2.1)$ & $17(14,22)$ & $3.4-5.6$ & $0.14,0.23,0.30,0.34,0.38$
\end{tabular}

TABLE 1. A summary of hydraulic conductivity, $k_{p}$, and absolute permeability, $K$, results obtained from permeability testing of the reticulated polyether foams. Lower and upper bounds of these estimates are shown in brackets. Also shown are lower and upper bounds for the size of the cells of the porous media, $l_{\text {cell }}$. Values of the permeability Reynolds number $R e_{K}$ used are also shown, which correspond to experiments conducted at the respective grid Reynolds numbers of $\operatorname{Re}_{G} \equiv M S f / \nu \approx 2020,4220,5260,6480$ and 8100 .

reticulated polyether foam that were inserted into the inner box, parallel to the grid, and overlaid up to a total thickness of $10 \mathrm{~cm}$. This ensured that the thickness of the permeable layers did not restrict the depth of flow penetration. The surface of the porous media was also located at a depth $H \approx 4.2 M$ below the grid's mean position.

Reticulated polyether foams have a regular structure comprising open cells that are pentagonal dodecahedra in shape (see, for example, Szycher 2012; Defonseka 2019). During the manufacturing process the thin membranes that initially form the faces of each cell are removed, such that the foams consist of a network of interconnected thin filaments. Consequently, reticulated polyether foams are $97 \%$ voids by volume (i.e. porosity of 97\%) (Szycher 2012; Defonseka 2019). The size of cells formed can be carefully controlled during the manufacturing process, thus foams are available over a wide range of permeabilities. For this study two foams of different permeabilities were used, which are shown in figure $1(\mathrm{c}, \mathrm{d})$. The geometry of these permeable media are ideal for studying the effects of permeability on wall turbulence as the high porosity and small filament thickness of the foams minimises the influence of the roughness of the interface between the porous layer and the fluid above. Consequently, reticulated polyether foams have also been used in previous studies investigating permeable boundaries (see, for example, Manes et al. 2011; Mujal-Colilles et al. 2015). The absolute permeability, $K$, of each foam used is shown in table 1 , which was determined using a constant head permeameter test (British Standards Institution 2010) in which the permeability was determined from measurements of the pressure drop across a sample at a given (constant) volume flow rate. Lower and upper bounds for the size of the dodecahedral cells, $l_{\text {cell }}$, present in each porous media, are also shown in table 1, which were provided by the manufacturer (Reticel, private communication). Cell sizes were determined using the Visiocell method (see, for example, Mullens et al. 2006, p. 236). Commercially, reticulated polyether foams are typically characterised by the mean number of pores present in a linear inch (PPI) of the permeable matrix; for this study 10PPI and 60PPI foams were used. We stress that this measure is poorly defined, since it is unclear whether in this context "pore" refers to the dodecahedral cells of the foam or the component faces of the cells. Consequently, this measure is used here only in a descriptive context in order to facilitate identification of similar foams used in previous studies. To ensure the foams were fully saturated when in use, each foam was submerged in a beaker of tap water and placed in a vacuum chamber to reduce the ambient pressure to approximately -0.9 Bar (gauge pressure), which deaerated the water and foam. The foams were thereafter kept submerged to prevent aeration of the foams.

For each boundary considered, we report results from 5 sets of experiments in which the 
stroke $S$ was set to be either $2.5 \mathrm{~cm}$ or $3.0 \mathrm{~cm}$ and the frequency of the grid's oscillation $f$ was varied between $1.6 \mathrm{~Hz}$ and $5.4 \mathrm{~Hz}$. The corresponding grid Reynolds numbers for these five experiments were $\operatorname{Re}_{G} \equiv M S f / \nu \approx 2020,4220,5260,6480$ and 8100 . For each experimental condition, the experiments were repeated, under nominally identical conditions, a total of 5 times; this approach facilitates the use of ensemble-averages to reduce scatter in the data. We note that we have also re-used measurements from previous experiments that investigated the interaction of oscillating-grid turbulence with an impermeable surface (McCorquodale \& Munro 2018a), but we focus here on reporting new data that illustrates the effects of boundary permeability on the interaction.

\subsection{Measurements and notation}

In each experiment, two-dimensional two-component PIV, applied to the vertical plane through the centre of the grid, as shown in figure 1(b), was used to acquire measurements of instantaneous fluid velocities in the region inside the inner box spanned by the grid and the permeable media. The flow was seeded with neutrally buoyant tracer particles (Pliolite with diameter range 75 to $125 \mu \mathrm{m}$ ), which were illuminated within a thin light sheet produced by a pulsed laser. Images of illuminated particles were recorded at 100 frames per second (at $1280 \times 1024$ pixel resolution) using a high-speed digital camera aligned perpendicular to the plane of the light sheet. PIV calculations were performed using square interrogation windows of $13 \times 13$ pixels, overlapped to achieve 8 pixel spacing between velocity vectors, resulting in a physical spacing between velocity vectors of approximately $0.16 \mathrm{~cm}$. We note that the parameters used for the PIV were chosen to conform with the guidelines recommended by Keane \& Adrian (1990).

The velocity data were calculated and analysed relative to the right-handed coordinate system $\left(x_{1}, x_{2}, x_{3}\right)$; here, $x_{3}$ denotes vertical depth below the mid-height of the grid's oscillation, and $\left(x_{1}, x_{2}\right)$ are the horizontal coordinates relative to the center of the grid (see figure 1$)$. The corresponding velocity components are denoted $\left(u_{1}, u_{2}, u_{3}\right)$; the two components measured using the PIV set-up described above are $u_{1}\left(x_{1}, x_{3}, t\right)$ and $u_{3}\left(x_{1}, x_{3}, t\right)$, in the central plane at $x_{2}=0$. We also introduce the coordinate $\xi=H-x_{3}$ to denote vertical height above the permeable boundary (see figure 1a). This coordinate is used only for convenience when plotting and comparing data; we stress that all velocities (and derivatives of velocities) were calculated in terms of the right-handed coordinates $\left(x_{1}, x_{2}, x_{3}\right)$. Sufficiently far beneath the grid (see section 2.3), oscillating-grid turbulence (OGT) is statistically stationary and so the statistical properties of the flow in this region were analysed using time averages. We use the conventional Reynolds decomposition $u_{i}=U_{i}+u_{i}^{\prime}$, where $u_{i}^{\prime}(\mathbf{x}, t)$ denote the fluctuating components and $U_{i}(\mathbf{x})=\overline{u_{i}}$ the time-averaged mean components (the overbar notation is used throughout to denote time averaging). In each experiment velocity data were captured for a period of $240 \mathrm{~s}$; analysis of the data showed that the time-averaged mean and rms of fluctuating velocity components were converged to within approximately $5 \%$ of their ultimate values over this time period (McCorquodale \& Munro 2018a,b).

Alongside measurement errors, the relatively slow convergence of the experimental data results in not-insignificant experimental uncertainty. However, we stress that the ensemble of 5 repeat tests for each experiment that we report provides an estimate of uncertainty within the data. In the analysis presented in sections 3 and 4 the experimental uncertainty is indicated by error bars. [A single representative set of error bars is shown in each figure to prevent the plots from becoming cluttered.] These error bars illustrate that the uncertainty in the experimental measurements is small in the context of permeability effects that we identify.

Finally, we note that the estimates of uncertainty described above do not include 
the influence of sampling errors arising from the limited resolution of the PIV data. That is, within the region of the flow for which velocity measurements were obtained (i.e. for $x_{3} \gtrsim 2.5 M$ ), the Kolmogorov length scale $\eta$ was estimated to be of the order $\eta \sim 0.05 \mathrm{~cm}$, which is finer than the physical spacing between velocity vectors computed by the PIV calculations (approx $0.16 \mathrm{~cm}$ ). [The Kolmogorov length scale was estimated using the relation $\eta=\nu^{3 / 4} \varepsilon^{-1 / 4}$ under the assumption that, in OGT, $\varepsilon \approx 0.75\left(\overline{u_{1}^{\prime 2}}\right)^{3 / 2} / \bar{\ell}$ for $x_{3} \gtrsim 2.5 M$ (Kit et al. 1997)]. Thus, the resolution used for PIV calculations was coarser than the smallest turbulent scales within the flow, such that velocity averaging occurred across interrogation windows and some turbulent fluctuations were unresolved. Consequently, the full energy content of the turbulent flow was not determined by the analysis. However, we stress that the range in scales of turbulent fluctuations that were under-resolved in the current analysis is very small in the context of the size of the integral scales of the turbulent flow (which are of the order $2 \mathrm{~cm}$ in size, see section 3 ). The implications of the limited resolution of the velocity measurements for the analysis presented in sections 3 and 4 is discussed within these sections.

\subsection{Description of the flow produced}

Close to the oscillating grid, henceforth referred to as the 'near-grid region', jets form in the wake of the grid elements resulting in a flow field characterised by the presence of energetic, mesh-sized coherent vortex structures that interact and breakdown as they are advected away from the grid. This coherent flow structure breaks down within a distance of 2.5 mesh lengths from the grid (i.e. for $x_{3} \lesssim 2.5 M$ ) (see, for example, McCorquodale \& Munro 2018b). Within the near-grid region the oscillation of the grid directly influences the structure of the flow on a time-scale on the order $1 / f$. However, under ideal conditions, the turbulent flow beyond this region, which we henceforth refer to as the 'turbulent-diffusive region', is statistically stationary, homogeneous and isotropic in planes parallel to the grid, with negligible mean flow (De Silva \& Fernando 1994). Moreover, velocity measurements in this region do not indicate the presence of periodic signatures relating to the grid forcing (McCorquodale \& Munro 2017). The turbulence is, however, inhomogeneous in planes normal to the grid; the r.m.s. turbulent velocity components $u$ and $w$ (where $w \equiv\left(\overline{u_{3}^{\prime} u_{3}^{\prime}}\right)^{1 / 2}, u \equiv\left(\overline{u_{1}^{\prime} u_{1}^{\prime}}\right)^{1 / 2}$ ) decay with increasing distance normal to the grid. The presence of the impermeable plate or porous layer inserted above the base of the acrylic box also results in a boundary-affected region of the flow, which we define as the thin layer of height $\delta_{s}$ above the boundary over which the degree of isotropy $w / u$ departs from a value of 1 and decreases as the boundary is approached. McCorquodale \& Munro (2017) reported that with the current apparatus $\delta_{s}$ is of the order of the integral length scale of the turbulence when the boundary is impermeable. The results reported here for permeable media are consistent with this observation (see section 3.1).

Under the idealised conditions assumed to occur beyond the near-grid region of the flow, the steady form of the Reynolds stress transport equations may be written as

$$
0=\underbrace{-\frac{\partial}{\partial x_{k}} \overline{u_{i}^{\prime} u_{j}^{\prime} u_{k}^{\prime}}}_{T_{i j}}-\underbrace{\frac{1}{\rho}\left(\frac{\partial}{\partial x_{i}} \overline{p^{\prime} u_{j}^{\prime}}+\frac{\partial}{\partial x_{j}} \overline{p^{\prime} u_{i}^{\prime}}\right)}_{\Pi_{i j}^{d}}+\underbrace{\frac{1}{\rho p^{\prime}\left(\frac{\partial u_{j}^{\prime}}{\partial x_{i}}+\frac{\partial u_{i}^{\prime}}{\partial x_{j}}\right)}}_{\Pi_{i j}^{s}}+\underbrace{\nu \frac{\partial^{2} \overline{u_{i}^{\prime} u_{j}^{\prime}}}{\partial x_{k} \partial x_{k}}}_{D_{i j}} \underbrace{-2 \nu \frac{\partial u_{i}^{\prime}}{\partial x_{k}} \frac{\partial u_{j}^{\prime}}{\partial x_{k}}}_{\varepsilon_{i j}} .
$$

The terms $T_{i j}$ and $\Pi_{i j}^{d}$ denote, respectively, transport by velocity and pressure fluctuations; $\Pi_{i j}^{s}$ is the inter-component energy redistribution due to the correlation between 
fluctuating strain and pressure fields; $D_{i j}$ and $\varepsilon_{i j}$ denote viscous diffusion and viscous dissipation. We stress that since the turbulence is approximately homogeneous on horizontal planes then $\overline{u_{i}^{\prime} u_{j}^{\prime}} \approx 0$ for $i \neq j$. Hence, a comprehensive understanding of the flow follows from considering terms of the transport equations for the Reynolds stresses $\overline{u_{1}^{\prime} u_{1}^{\prime}}, \overline{u_{2}^{\prime} u_{2}^{\prime}}$ and $\overline{u_{3}^{\prime} u_{3}^{\prime}}$ (i.e. $u^{2}, v^{2}$ and $w^{2}$ ), and the transport equation for TKE, which is obtained from the trace of $(2.1)$ noting that $\overline{u_{i}^{\prime} u_{i}^{\prime}} / 2=\overline{q^{\prime 2}}$ denotes the TKE.

From (2.1) it follows that the TKE transport equation may be written as

$$
0=\underbrace{-\frac{1}{2} \frac{\partial}{\partial x_{k}} \overline{u_{i}^{\prime} u_{i}^{\prime} u_{k}^{\prime}}}_{T_{i i}}-\underbrace{-\frac{1}{\rho} \frac{\partial}{\partial x_{i}} \overline{p^{\prime} u_{i}^{\prime}}}_{\Pi_{i i}^{d}}+\underbrace{\frac{\nu}{2} \frac{\partial^{2} \overline{u_{i}^{\prime} u_{i}^{\prime}}}{\partial x_{k} \partial x_{k}}}_{D_{i i}} \underbrace{-\nu \frac{\overline{\partial u_{i}^{\prime}}}{\partial x_{k}} \frac{\partial u_{i}^{\prime}}{\partial x_{k}}}_{\varepsilon_{i i}},
$$

but by noting that turbulence is homogeneous in the $x_{1}-x_{2}$ plane, parallel to the grid, such that turbulence statistics only vary in the $x_{3}$ direction, the TKE budget can be simplified to

$$
0=-\frac{\mathrm{d}}{\mathrm{dx}_{3}}\left(\overline{\frac{p^{\prime} u_{3}^{\prime}}{\rho}}+\overline{u_{3}^{\prime} q^{\prime 2}}+\nu \frac{\mathrm{d}}{\mathrm{dx}_{3}} \overline{q^{\prime 2}}\right)+\varepsilon .
$$

Outside the boundary-effected region the viscous transport term can be assumed to be negligible, since the transfer process is predominantly inertial in high Reynolds number flows, and thus in the turbulent-diffusive region the flow is governed by a balance of the viscous dissipation of TKE and the transport of TKE by velocity and pressure fluctuations.

By parametrising the leading order terms of equation (2.3), Thompson \& Turner (1975) and Hopfinger \& Toly (1976) were able to obtain an expression describing the spatial decay of the r.m.s. velocity components $u$ and $w$ with increasing distance normal to the grid, valid within the turbulent-diffusive region. The resulting expression has since been validated empirically and flow in the turbulent-diffusive region is commonly described by the standard model

$$
\begin{gathered}
u=C_{1} S f\left(\frac{x_{3}}{M^{1 / 2} S^{1 / 2}}\right)^{-\gamma}, \\
w=C_{2} u,
\end{gathered}
$$

with $\gamma \approx 0.8-1.5, C_{1} \approx 0.2-0.5$ and $C_{2} \approx 1.1-1.4$ (Thompson \& Turner 1975; Hopfinger \& Toly 1976; McDougall 1979; Hopfinger \& Linden 1982; Atkinson et al. 1987; Nokes 1988; De Silva \& Fernando 1994; Kit et al. 1997).

Simple expressions can also be obtained to describe how the time-averaged mean dynamic pressure, denoted $P$, should vary within the turbulent-diffusive and boundaryeffected regions of the flow. That is, rearranging the steady mean-flow momentum equations, and omitting the body force term for gravitational acceleration since we concerned with the dynamic pressure, gives

$$
\frac{\partial P}{\partial x_{i}}=-\rho U_{j} \frac{\partial U_{i}}{\partial x_{j}}-\rho \frac{\partial\left(\overline{u_{i}^{\prime} u_{j}^{\prime}}\right)}{\partial x_{j}}+\mu \frac{\partial^{2} U_{i}}{\partial x_{j} \partial x_{j}},
$$

where $\mu$ denotes dynamic viscosity. Under ideal conditions (i.e. negligible mean flow, with turbulence that is homogeneous in the $x_{1}-x_{2}$ plane, parallel to the grid), these equations simplify to

$$
\frac{\partial P}{\partial x_{3}}=-\rho \frac{\partial\left(\overline{u_{3}^{\prime} u_{3}^{\prime}}\right)}{\partial x_{3}}=-\rho \frac{\partial w^{2}}{\partial x_{3}} \quad \text { and } \quad \frac{\partial P}{\partial x_{i}}=0, i=1,2
$$


Hence, in light of equation (2.4), within the turbulent diffusive region we expect the mean dynamic pressure to increase with depth beneath the grid, but at a rate that decays with increasing $x_{3}$.

In practice, minor differences in the flow produced by OGT apparatus are found to occur relative to the idealised flow described above. In particular, OGT apparatus are known to exhibit secondary circulations (see, for example, McKenna \& McGillis 2004), which give rise to small mean flow velocities within the turbulent-diffusive and boundaryeffected regions of the flow. We stress that the OGT apparatus used in this study has been specifically designed to conform with experimental conditions that have been found to minimise the magnitude of the mean flow velocities within the turbulent-diffusive and boundary-effected regions of the flow (Hopfinger \& Toly 1976; McDougall 1979; Fernando \& De Silva 1993; McCorquodale \& Munro 2018b). Consequently, previous studies using the same apparatus (McCorquodale \& Munro 2017, 2018b) indicate that within the turbulent-diffusive and boundary-effected region of the flow the turbulent velocity components are of comparable or greater magnitude than mean flow velocity components. Moreover, having acquired measurements of terms in the transport equation for TKE representing the transport and production due to the mean flow, McCorquodale \& Munro (2017) concluded that although the presence of a mean flow indicates the presence of mean shear in the boundary-effected region - such that the turbulence is not strictly zero-mean shear - the levels are sufficiently small in magnitude to allow meaningful comparisons to be made with zero-mean-shear conditions. McCorquodale \& Munro $(2017,2018 b)$ also showed that anisotropic regions exist adjacent to the tank sidewalls, but that the flow in the turbulent-diffusive region is approximately homogeneous on the $x_{1}-x_{2}$ plane, parallel to the grid, over a central region of the inner tank (i.e. for $\left|x_{1} / L\right| \leqslant 1 / 2$ ). Hence throughout this paper our attention is focused on the central region $\left|x_{1} / L\right| \leqslant 1 / 2$ and the sidewall anisotropic regions are ignored in the calculation of turbulent statistics. The notation $\langle\cdot\rangle_{1}$ is henceforth used to denote quantities that have been spatially averaged, in the $x_{1}$ direction, over this region.

For each experiment reported here, data describing the statistical structure of the mean and turbulent components of the flow above the boundary-effected region were in good agreement with the above description of the flow. [We note that representative results describing the structure of the flow produced by the apparatus have also been reported previously by McCorquodale \& Munro (2017) and McCorquodale \& Munro (2018b).] Consequently, here we focus on reporting results within the boundary-effected region of the flow, which, recall, we define as the thin layer of height $\delta_{s}$ above the permeable boundary over which the degree of isotropy $w / u$ departs from a value of 1 and decreases as the boundary is approached.

\subsection{Permeability Reynolds number}

Throughout this paper the effects of boundary permeability are characterised using the permeability Reynolds number $R e_{K}$, which is a measure of the inhibiting effects of viscous forces at a permeable boundary. Physically, $R e_{K}$ can be interpreted as the ratio of the typical pore size in a permeable matrix, which scales with the square root of the absolute permeability $\sqrt{K}$ (see for example Katz \& Thompson 1986), to the typical viscous sublayer thickness $\delta_{v}$ over the surface of the elements that constitute the permeable medium. [The viscous sublayer at the surface of a porous media retains the same interpretation as the viscous sublayer at an impermeable boundary; the viscous sublayer is the region of flow adjacent to the surface(s) of an impermeable boundary or a porous media in which viscous stresses predominate over turbulent stresses.] In a channel 
flow the viscous sublayer scales with $\nu / u_{*}$, such that (Breugem et al. 2006)

$$
R e_{K} \equiv \frac{u_{*} \sqrt{K}}{\nu},
$$

where $u_{*}$ denotes the friction velocity.

However, an alternative expression for the viscous sublayer thickness is required for the current problem in which zero-mean-shear interacts with a boundary $\left[u_{*}\right.$ is undefined in the current flow]. In studying the interaction of an initially isotropic turbulent flow with a impermeable surface that moves at the free-stream velocity of the turbulent flow, such that there is zero-mean-shear in the boundary-effected region of the flow, Hunt \& Graham (1978) proposed that the viscous sublayer thickness $\delta_{v}$ over the impermeable boundary scaled as

$$
\delta_{v} \propto\left[\nu \ell / u_{\delta}\right]^{1 / 2}
$$

where $\ell$ denotes the integral length scale of the turbulence outside the boundaryeffected region and $u_{\delta}=\left(\overline{u_{1}^{\prime} u_{1}^{\prime}}\right)^{1 / 2}$ at $\xi=\delta_{s}$. Since these terms are defined at the edge of the boundary-effected region, these definitions preclude any effects on the flow that arise from unknown effects relating to the modifying effects of the boundary. In addition, in turbulence that is otherwise isotropic outside the boundary-affected region $\overline{u_{1}^{\prime} u_{1}^{\prime}}=\overline{u_{2}^{\prime} u_{2}^{\prime}}=\overline{u_{3}^{\prime} u_{3}^{\prime}}$ so that each velocity component can be used interchangeably for this characteristic value of $u_{\delta}$. Hunt (1984) subsequently asserted the validity of this expression for the flow considered in this study, in which statistically steady turbulence interacts with a surface in the absence of mean shear. That is, in the current flow, $\delta_{v}$ is estimated to be smaller than the integral length scale $\ell$, which scales with the thickness of the boundary-effected region, by a factor equal to the square root of the turbulent Reynolds number, i.e. $\delta_{v} / \delta_{s} \propto \delta_{v} / \ell \propto R e^{-1 / 2}$ where $R e \equiv u_{\delta} \ell / \nu$. Equation (2.8) gives rise to a permeability Reynolds number defined as

$$
R e_{K} \equiv \sqrt{\frac{u_{\delta} K}{\nu \ell}}
$$

The values of $R e_{K}$ for each experimental condition, evaluated using (2.9), are shown in table 1 .

We note that although previous studies using OGT have reported measurements of the viscous sublayer thickness of the order given by equation (2.8) (Brumley \& Jirka 1987; Kit et al. 1997), here the corresponding measurements did not obey the implied $R e^{-1 / 2}$ scaling. That is, equation (2.8) predicts values of $\delta_{v}$ of the correct order of magnitude, but the measurements of $\delta_{v}$ did not exhibit any consistent Reynolds number scaling. We attribute this result to the small Reynolds number range considered and uncertainty in the methods used to define the edge of the viscous sublayer.

\section{Statistical structure of turbulence in the boundary-affected region}

In this section we present experimental results to show how boundary permeability affected measurements of the r.m.s velocity components, vertical flux of TKE and mean dynamic pressure gradient, which provide evidence of the mechanisms governing the interaction. We note that, since we were unable to fully resolve the dissipative scales within the flow (see section 2.1), this may lead to a slight underestimate of the total energy content within the flow. However, we stress that in this section we are primarily concerned with the effect of the boundary on the (well-resolved) large scales within the flow, such that this limitation does not alter the conclusions drawn. 

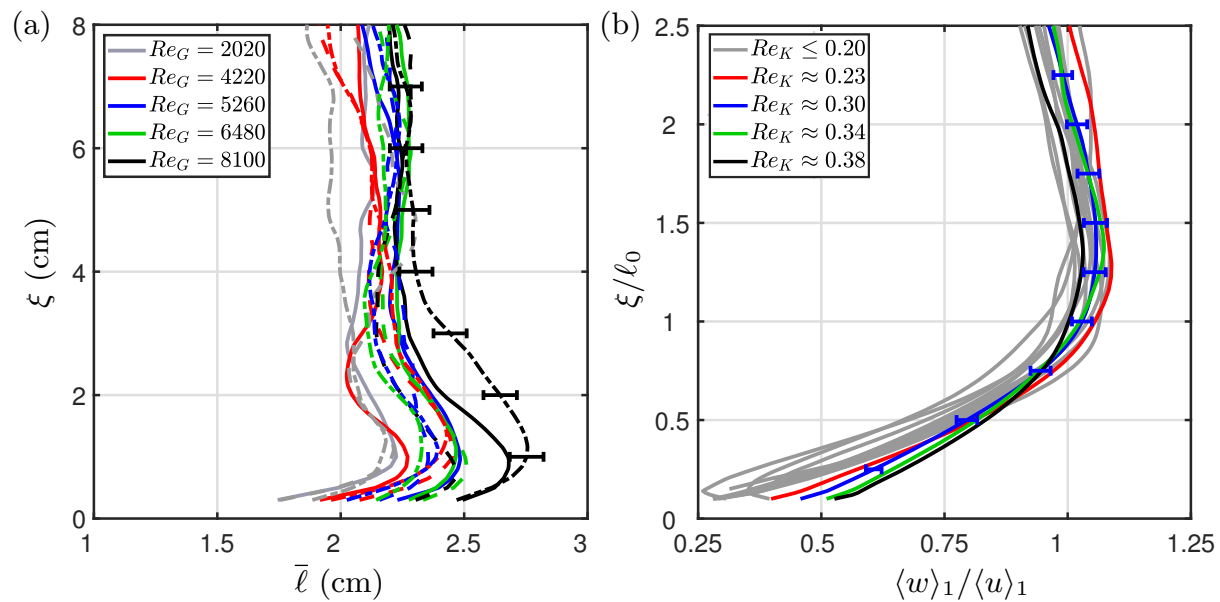

Figure 2. (a) Computed values of the time-averaged integral length scale, $\bar{\ell}$, plotted against height $\xi$. (b) Measurements of the degree of isotropy $\langle w\rangle_{1} /\langle u\rangle_{1}$, plotted against normalised height $\xi / \ell_{0}$. In both plots a single data-set is shown for each experimental condition reported in table 1 , which is an average of the measurements obtained across the $n=5$ repeats conducted for each condition. In (a) data obtained at different $R_{G}$ are shown separately by linecolour [see legend], whilst the type of boundary in use is shown by the linetype; '-', '--' and '...' denote an impermeable surface, 60 ppi porous layer and 10 ppi porous layer respectively. In (b) data at different $R e_{K}$ are shown separately, irrespective of $R e_{G}$ [see legend]. Also shown are representative error bars, corresponding to the standard error across the $n=5$ repeats; error bars are shown for the cases $\operatorname{Re}_{G} \approx 8100$ and $R e_{K} \approx 0.30$ in (a) and (b) respectively.

\subsection{Thickness of the boundary-affected region}

In section 2 we defined the boundary-effected region as a thin layer, of thickness $\delta_{s}$, directly above the boundary over which the degree of isotropy $\langle w\rangle_{1} /\langle u\rangle_{1}$ departs from its value of approximately 1 away from the boundary, and decreases as the boundary is approached. At this point it is instructive to define a reference value $\ell_{0}$ of the (timeaveraged) integral length scale $\bar{\ell}$; previous research indicates that $\delta_{s}$ scales with the integral length scale of the turbulence (Perot \& Moin 1995).

Estimates for the integral length scales were obtained from the velocity measurements by computation of autocorrelation coefficients, using the approach previously described by Kit et al. (1997) and McCorquodale \& Munro (2017). That is, the integral length scale $\ell$ is defined as the integral of the autocorrelation function of $u_{1}^{\prime}\left(x_{1}, x_{3}, t\right)$, over the spatial lag up to which the autocorrelation function first crosses zero; time-averaged integral length scales are denoted $\bar{\ell}$. The computed values of $\bar{\ell}$ are shown in figure 2(a), plotted against height, $\xi$, above the boundary. Figure 2(a) shows that $\bar{\ell}$ exhibits a notable degree of scatter, but for $\xi \gtrsim 3 \mathrm{~cm}$ the data are relatively constant, taking values typically between $2 \mathrm{~cm}$ and $2.3 \mathrm{~cm}$. For heights $\xi<3 \mathrm{~cm}$ the values of $\bar{\ell}$ increase slightly, before rapidly reducing at $\xi \approx 0.75 \mathrm{~cm}$. Figure $2($ a) also shows that slightly larger values of $\bar{\ell}$ are obtained for experiments conducted at the larger values of $R e_{G}$ considered. No link between the values of $\bar{\ell}$ and permeability Reynolds number $R e_{K}$ was identified. In order to facilitate comparison with previous work (McCorquodale \& Munro 2017, 2018a), we define the reference integral length scale $\ell_{0}$ to be the peak value attained by $\bar{\ell}$ in the near-boundary region.

Turning now to estimates of the thickness of the boundary-affected region, measured values of the degree of isotropy $\langle w\rangle_{1} /\langle u\rangle_{1}$ are shown in figure 2(b), plotted against scaled height $\xi / \ell_{0}$. Figure $2(\mathrm{~b})$ shows a rapid increase in anisotropy occurs at $\xi / \ell_{0} \approx 1$ as the 
boundary is approached, departing from the far-field trend $\langle w\rangle_{1} /\langle u\rangle_{1} \approx 1$. No link has been identified with either $R e_{G}$ or $R e_{K}$ and the point at which this departure from the far-field trend occurs. We therefore conclude that the boundary-effected region extends up to $\delta_{s} / \ell_{0} \approx \xi / \ell_{0} \approx 1$ across the entire $R e_{K}$ range considered.

However, we note that the data in figure 2(b) show the degree of anisotropy over the region $\xi / \ell_{0} \lesssim 0.5$ is affected by the boundary permeability $R e_{K}$. That is, increasing $R e_{K}$ reduces the observed anisotropy. In figure $2(\mathrm{~b})$, results for $R e_{K} \lesssim 0.2$ collapse, to within experimental uncertainty, onto the data obtained for $R e_{K} \approx 0$, but there is a distinct departure from this trend for $R e_{K} \gtrsim 0.2$. This indicates that for $R e_{K} \lesssim 0.2$ the boundary is effectively impermeable and the no-penetration and viscous boundary conditions are, at least approximately, enforced. In contrast, for $R e_{K} \gtrsim 0.2$ boundary permeability has a contributing effect to the turbulence structure in the boundary-effected region.

\subsection{Root-mean-square velocity data and Turbulent kinetic energy}

Here, we consider the rms of fluctuating velocity components in more detail in order to explain how boundary permeability influences the degree of isotropy within the boundaryeffected region. The effect of the boundary on the rms turbulent velocity components is shown in figure $3(\mathrm{a}, \mathrm{b})$. The data have been normalised using values of the rms turbulent velocity components that we would expect in the absence of the boundary. That is, values of $u$ and $w$ that would be expected in the absence of the boundary, which we denote $u_{0}$ and $w_{0}$, were determined by applying a best fit of the form given by equation (2.4) to measurements of $\langle u\rangle_{1}$ and $\langle w\rangle_{1}$ in the turbulent-diffusive region of the flow (i.e. $\xi>\ell_{0}$ ) and extrapolating the best fit to the boundary-affected region of the flow (i.e. $\xi<\ell_{0}$ ). Using this approach, estimates of $u_{0}$ and $w_{0}$ were evaluated for each vertical location within the boundary-affected region of the flow at which measurements of $\langle u\rangle_{1}$ and $\langle w\rangle_{1}$ were obtained. A similar procedure has also been used to obtain reference values for other parameters reported in this section.

Figure 3(a) shows that the boundary-normal component $w$ is monotonically reduced by the presence of the boundary. For $R e_{K} \lesssim 0.2$ the observed reduction in $w$ is in quantitative agreement with results from previous studies investigating the interaction of zero-mean-shear turbulence with an impermeable boundary (Hunt \& Graham 1978; Thomas \& Hancock 1977; Hannoun et al. 1988; Aronson et al. 1997). However, figure 3 (a) also shows that the magnitude of $w / w_{0}$ over $\xi / \ell_{0} \lesssim 0.5$ is slightly greater for experiments conducted at $R e_{K}>0.2$ and suggests the magnitude increases with increasing $R e_{K}$. Although a small effect, these data indicate that for $R e_{K} \gtrsim 0.2$ the boundary permeability begins to inhibit the kinematic blocking condition that is otherwise enforced when $R e_{K}=0$.

A more pronounced $R e_{K}$ effect is evident in the measurements of the horizontal (i.e. boundary-tangential) component $(u)$, shown in figure $3(\mathrm{~b})$. The results indicate that for $R e_{K} \lesssim 0.2$ the boundary acts to increase $u$ within the boundary-affected region, relative to the far-field trend, except in a thin viscous region immediately adjacent to the boundary. These data are in agreement with results from previous studies investigating the interaction of OGT, and random jet-arrays, with an impermeable boundary (Brumley \& Jirka 1987; Hannoun et al. 1988; McCorquodale \& Munro 2017; Johnson \& Cowen 2018). Notably, however, figure $3(\mathrm{~b})$ shows that the amplification of $u / u_{0}$ within the boundary-effected region $\left(\xi / \ell_{0} \lesssim 1\right)$ is significantly inhibited with increasing $R e_{K}$, for $R e_{K} \gtrsim 0.2$.

Given this relative increase in $w$ and relative decrease in $u$, within the boundaryaffected region, with increasing $R e_{K}$, a logical explanation would be that changes in intercomponent energy transfer between the velocity components occur as the boundary 

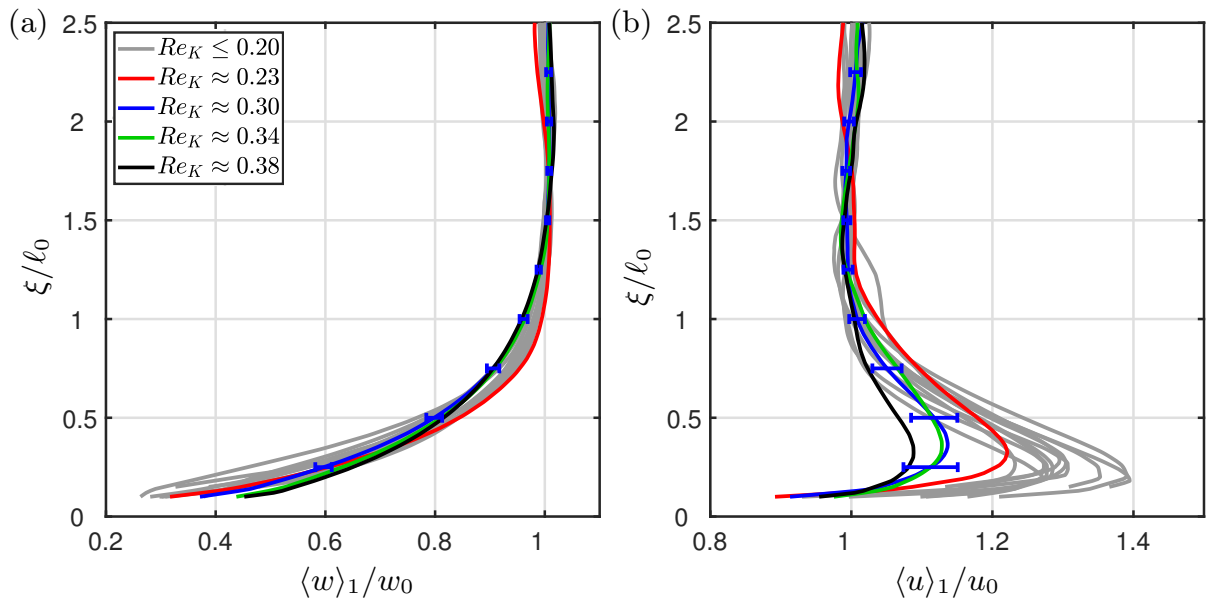

FiguRE 3. Plots showing the scaled rms of fluctuating velocity components (a) $\langle w\rangle_{1} / w_{0}$ and (b) $\langle u\rangle_{1} / u_{0}$. In each plot a single data-set is shown for each experimental condition reported in table 1 , which is an average of the measurements obtained across the $n=5$ repeats conducted for each condition. The data at different $R e_{K}$ are shown separately, irrespective of $R e_{G}$. Also shown are representative error bars for the case $R e_{K} \approx 0.3$, corresponding to the standard error across the $n=5$ repeats.

permeability increases. Recall, intercomponent energy transfer is described by the term $\Pi_{i j}^{s}$ in equation 2.1. Indeed, in section 4 we argue that this is one of the contributing mechanisms. However, the changes in the velocity components described above also correspond to changes in turbulent kinetic energy within the boundary-effected region.

Measurements of TKE, here defined as $\overline{q^{\prime 2}}=\overline{u_{i}^{\prime} u_{i}^{\prime}} / 2 \approx\left(2 \overline{u_{1}^{\prime} u_{1}^{\prime}}+\overline{u_{3}^{\prime} u_{3}^{\prime}}\right) / 2$ (where $v \approx u$ has been assumed), are shown in figure 4(a). In accordance with the results of the rms turbulent velocity components, figure 4 (a) shows $\left\langle\overline{q^{\prime 2}}\right\rangle_{1}$ scaled by $\overline{q_{0}^{\prime 2}}$, which denotes the values of $\left\langle\overline{q^{\prime 2}}\right\rangle_{1}$ that would be expected in the absence of the boundary, obtained by extrapolating a best fit applied to the data above the boundary-affected region. Figure 4(a) shows that when $R e_{K} \lesssim 0.2$ (i.e. the boundary is effectively impermeable) the results exhibit $\left\langle\overline{q^{\prime 2}}\right\rangle_{1} / \overline{q_{0}^{\prime 2}}>1$ within the boundary-affected region, in accordance with previous results at an impermeable boundary (Hannoun et al. 1988; McCorquodale \& Munro 2017; Johnson \& Cowen 2018). This indicates that for $R e_{K} \lesssim 0.2$ there is an increase in turbulent kinetic energy within the boundary-affected region $\left(\xi / \ell_{0} \lesssim 1\right)$, relative to expected values in the absence of the boundary. However, figure 4(a) shows significant reductions in $\left\langle\overline{q^{\prime 2}}\right\rangle_{1} / \overline{q_{0}^{\prime 2}}$ occur within the boundary-affected region as $R e_{K}$ is increased. Moreover, for $R e_{K} \approx 0.38$ figure $4\left(\right.$ a) shows that $\left\langle\overline{q^{\prime 2}}\right\rangle_{1} / \overline{q_{0}^{\prime 2}}<1$ when $\xi / \ell_{0}<1$, indicating that in this case there is no increase in turbulent kinetic energy within the boundary-affected region, relative to expected values in the absence of the boundary. This reduction in TKE within the boundary-affected region (relative to an impermeable boundary) indicates that the changes in $u / u_{0}$ and $w / w_{0}$ described above cannot be explained only by changes in intercomponent energy transfer. Instead, the results can be explained using measurements of the TKE flux.

A TKE flux exists throughout the flow due to the vertically inhomogeneous nature of the flow produced by OGT, whereby the magnitude of turbulent fluctuations decays with distance beneath the grid resulting in a flux of TKE vertically downwards and away from the grid. In section 2.3 we emphasised that the transport of TKE by velocity fluctuations $T_{i i}$ is a key process in determining the statistical structure of the flow in zero-mean-shear 

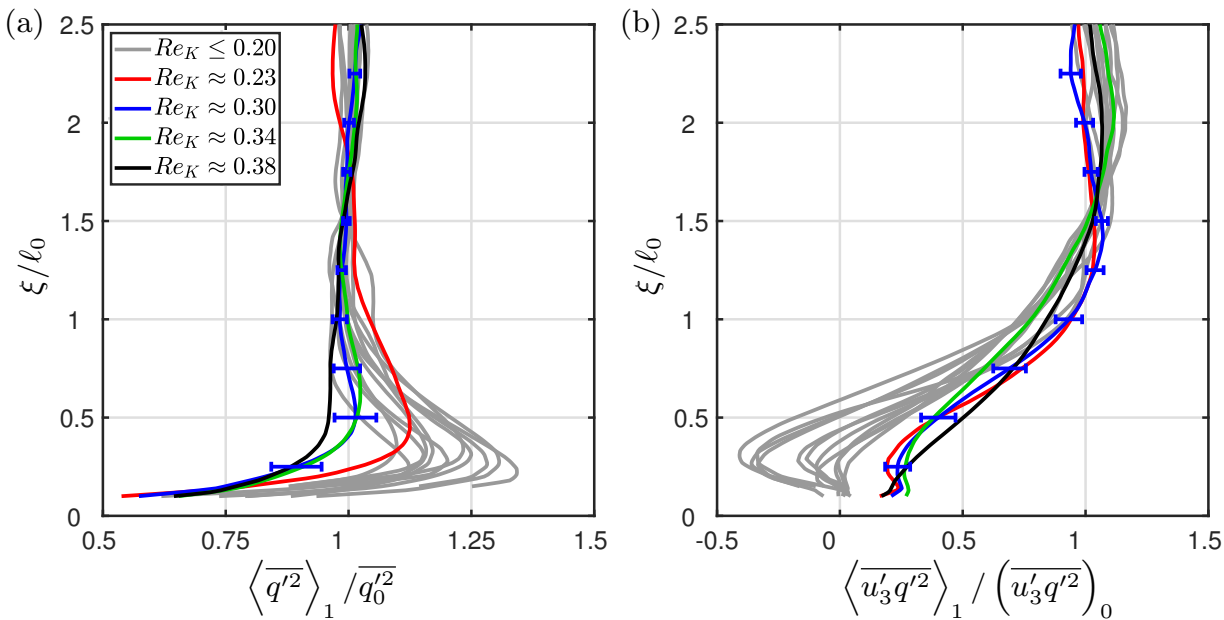

Figure 4. Measurements of (a) TKE $\left\langle\overline{q^{\prime 2}}\right\rangle_{1}$, and (b) vertical flux of TKE, $\left\langle\overline{u_{3}^{\prime} q^{\prime 2}}\right\rangle_{1}$. Here, $\left\langle\overline{q^{\prime 2}}\right\rangle_{1}$ and $\left\langle\overline{u_{3}^{\prime} q^{\prime 2}}\right\rangle_{1}$ have been normalised by the values expected in the absence of the boundary, denoted $\overline{q_{0}^{\prime 2}}$ and $\left(\overline{u_{3}^{\prime} q^{\prime 2}}\right)_{0}$ respectively. In both plots a single data-set is shown for each experimental condition reported in table 1 , which is an average of the measurements obtained across the $n=5$ repeats conducted for each condition. The data at different $R e_{K}$ are shown separately, irrespective of $R e_{G}$. Also shown are representative error bars for the case $R e_{K} \approx 0.3$, corresponding to the standard error across the $n=5$ repeats.

turbulence; the TKE transport equation for this flow is $0=T_{i i}+\Pi_{i i}^{d}+D_{i i}+\varepsilon_{i i}$, where $T_{i i}=-\mathrm{d}\left(\overline{u_{3}^{\prime} q^{\prime 2}}\right) / \mathrm{d} x_{3}$. Measurements of the vertical flux of TKE, $\left\langle\overline{u_{3}^{\prime} q^{\prime 2}}\right\rangle_{1}$, are shown in figure $4(\mathrm{~b})$. As before, the data have been scaled by their expected trend in the absence of the boundary, denoted $\left(\overline{u_{3}^{\prime} q^{\prime 2}}\right)_{0}$, again obtained from a best fit applied to the data above the boundary-affected region. Previous studies of OGT interacting with an impermeable boundary reported a reduction in the TKE flux within the boundary-affected region $\left(\xi / \ell_{0} \lesssim 1\right)$ relative to the far-field (McCorquodale \& Munro 2017; Hannoun et al. 1988) as a result of the kinematic blocking effect of the boundary. In terms of the TKE transport equation, this observation corresponds to an increase in the transport of TKE by velocity fluctuations $T_{i i}$ within the boundary-affected region, which is offset by a reduction in transport of TKE by pressure fluctuations $\Pi_{i i}^{d}$; within the boundary-affected region, values of $\varepsilon_{i i}$ and $D_{i i}$ have been reported to be approximately constant except within the viscous sublayer (McCorquodale \& Munro 2017).

For $R e_{K} \lesssim 0.2$ the data in figure 4(b) are consistent with the trend previously reported for the case of an impermeable boundary and reveal that for $\xi / \ell_{0} \lesssim 0.5$ the direction of energy flux is reversed $\left(\right.$ i.e. $\left.\left\langle\overline{u_{3}^{\prime} q^{\prime 2}}\right\rangle_{1} /\left(\overline{u_{3}^{\prime} q^{\prime 2}}\right)_{0}<0\right)$, such that there is a small flux of energy away from the boundary. The result of this blocking of the TKE flux within the boundaryaffected region is an increase in TKE (McCorquodale \& Munro 2017; Hannoun et al. 1988), as shown in figure 4(a), since additional energy that would otherwise propagate past the boundary (in its absence) is trapped in the boundary-affected region. Thus, this effect gives rise to an increase in the boundary-tangential velocity components relative to expected values in the absence of the boundary (McCorquodale \& Munro 2017), as shown in figure $3(\mathrm{~b})$. We note that McCorquodale \& Munro (2018a) also proposed that, for $R e_{K}=0$, a weak net intercomponent energy transfer from $w^{2}$ to $u^{2}$ occurs over the region $0.3 \lesssim \xi / \ell_{0} \lesssim 0.6$; this is consistent with the reversed direction of TKE flux shown in figure 4(b) over this region, and also contributes to observed values of $u / u_{0}>1$.

At a permeable boundary (i.e. for $R e_{K} \gtrsim 0.2$ ) the data in figure 4(b) indicate that 

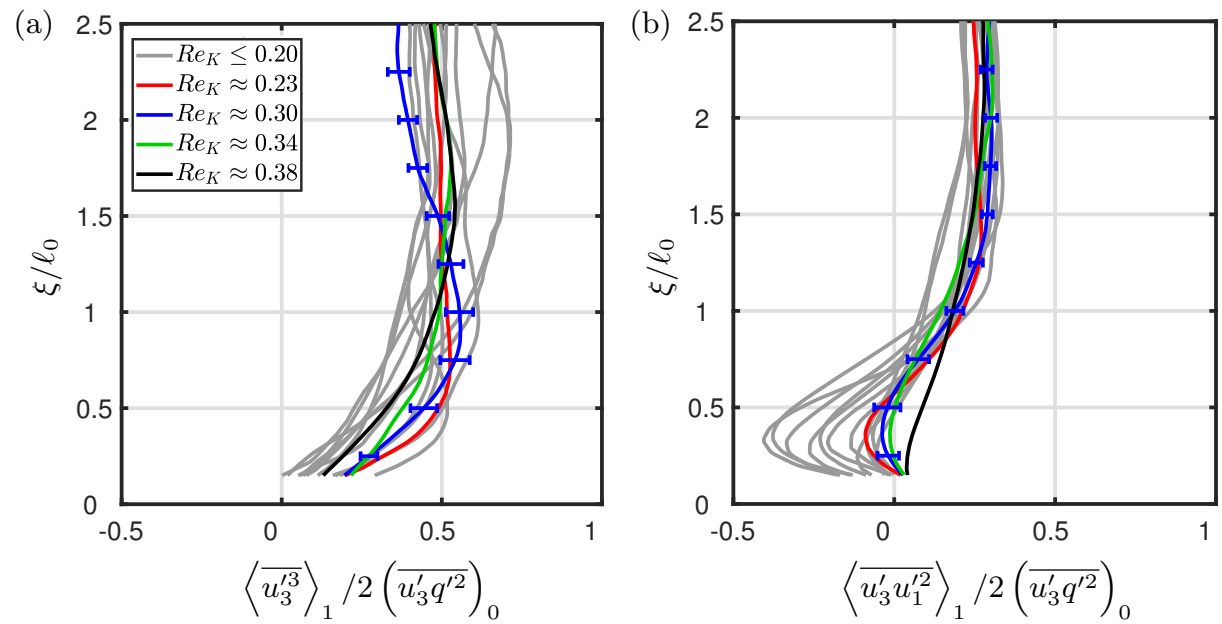

Figure 5. Measurements of the components of the TKE flux (a) $\left\langle\overline{u_{3}^{\prime 3}}\right\rangle_{1}$ and (b) $\left\langle\overline{u_{1}^{\prime 2} u_{3}^{\prime}}\right\rangle_{1}$, plotted against scaled height $\xi / \ell_{0}$. Each component has been normalised by the values for the total TKE flux expected in the absence of the boundary denoted $\left(\overline{u_{3}^{\prime} q^{\prime 2}}\right)_{0}$. In both plots a single data-set is shown for each experimental conditions reported in table 1 , which is an average of the measurements obtained across the $n=5$ repeats conducted for each condition. The data at different $R e_{K}$ are shown separately, irrespective of $R e_{G}$. Also shown are representative error bars for the case $R e_{K} \approx 0.3$, corresponding to the standard error across the $n=5$ repeats.

the vertical energy flux does not reduce by as much within the boundary-affected region $\left(\xi / \ell_{0}<1\right)$ relative to experiments at an effectively impermeable boundary $\left(R e_{K} \lesssim 0.2\right)$. This trend indicates that the amplification of $T_{i i}$ within the boundary-affected region $\left(\xi / \ell_{0} \lesssim 1\right)$ is inhibited with increasing $R e_{K}$, for $R e_{K} \gtrsim 0.2$. Most importantly, these data show that as the boundary is approached the TKE flux remains positive and so is oriented into the porous media. This increase in energy flux is attributed to a reduction in the blocking effect of the boundary (consistent with figure $3 \mathrm{a}$ ) which enables a weak flux of energy into the permeable medium when $R e_{K} \gtrsim 0.2$.

To better understand the vertical flux of TKE into the boundary-affected region we have decomposed $\left\langle\overline{u_{3}^{\prime} q^{\prime 2}}\right\rangle_{1}$ into its components $\left.\left\langle\overline{u_{3}^{\prime} u_{1}^{\prime 2}}\right\rangle_{1}, \overline{\left\langle u_{3}^{\prime 3}\right.}\right\rangle_{1}$, which are shown in figure 5 against scaled height $\xi / \ell_{0}$.

The vertical flux of $w^{2}$ is shown in figure $5(\mathrm{a})$, and is positive far from the boundary, which indicates a flux of energy away from the grid transported by turbulent fluctuations.

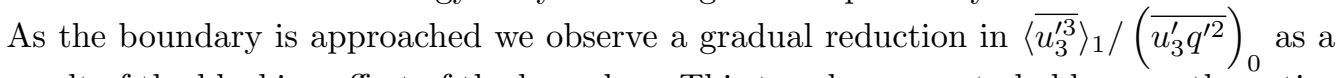
result of the blocking effect of the boundary. This trend appears to hold across the entire $R e_{K}$ range considered here, which is consistent with the results shown in figure 3(a) in which we observed that only very small increases in $w / w_{0}$ occurred as $R e_{K}$ increased.

The vertical flux of $u^{2}$ is shown in figure $5(\mathrm{~b})$, and is also positive far from the boundary, indicating a flux of energy away from the grid. When $R e_{K} \lesssim 0.2,\left\langle\overline{u_{3}^{\prime} u_{1}^{\prime 2}}\right\rangle_{1}$ decreases on approach to the boundary, reaching approximately 0 at $\xi / \ell_{0} \approx 0.6$. This trend can be attributed to the blocking effect of the boundary on the vertical TKE flux, which in isolation would act to give rise to constant values of $u$ within the boundary-affected region and correspondingly zero net vertical energy flux. For $\xi / \ell_{0} \lesssim 0.6$ the vertical flux of $u^{2}$ is small but becomes negative indicating that there is a small net flux of $u^{2}$ away from the boundary, which is associated with a net intercomponent energy transfer from $w^{2}$ to $u^{2}$ as fluid elements are blocked by the surface (McCorquodale \& Munro 2018a). 
This trend continues until $\xi / \ell_{0} \approx 0.3$, at which point $\left\langle\overline{u_{3}^{\prime} u_{1}^{\prime 2}}\right\rangle_{1}$ tends to zero as a result of viscous dissipative effects that are prevalent over the viscous sublayer (McCorquodale \& Munro 2017). [We note that the viscous sublayer (in which significant dissipation of TKE occurs) has thickness of approximately 0.2 to $0.3 \ell_{0}$ (see figure $4 \mathrm{a}$ )]. The negative values of $\left\langle\overline{u_{3}^{\prime} u_{1}^{\prime 2}}\right\rangle_{1}$ for $\xi / \ell_{0} \lesssim 0.6$ are responsible for the small negative values of $\overline{u_{3}^{\prime} q^{\prime 2}}$ observed over the region $\xi / \ell_{0} \lesssim 0.5$ in figure $4(\mathrm{~b})$.

When $R e_{K} \gtrsim 0.2$, results shown in figure $5(\mathrm{~b})$ show that $\left\langle\overline{u_{3}^{\prime} u_{1}^{\prime 2}}\right\rangle_{1}$ is not reduced by as much within the boundary-affected region and, at the largest $R e_{K}$, retains a positive value throughout, indicating that there is a weak net flux of $u^{2}$ into the porous media. This result indicates that there is reduction in the blocking of the far-field energy flux as $R e_{K}$ increases and is consistent with the observed reduction in $u / u_{0}$ when $R e_{K} \gtrsim 0.2$ (figure 3c).

To summarise, results of the TKE and vertical flux of TKE indicate that a contributing factor to the reported reduction in $u / u_{0}$ as $R e_{K}$ increases (see figure $3 \mathrm{~b}$ ) is a reduction in the blocking effect of the boundary on the vertical TKE flux, giving rise to a net flux of $u^{2}$ into the porous media when $R e_{K} \gtrsim 0.2$. This reduction in the blocking effect of the boundary on the TKE flux gives rise to the reduction in TKE within the boundary-affected region, as shown in figure 4(a), as energy is no longer trapped in the boundary-affected region. Note, however, that although the kinematic blocking condition is inhibited for $R e_{K} \gtrsim 0.2$, viscous dissipation is still significant close to the surface.

Further insight into the effects of boundary permeability can be derived by considering the time-averaged mean dynamic pressure, denoted $P$. To estimate the vertical meanpressure gradient, $\partial P / \partial x_{3}$, in the boundary-affected region we used the measured velocity data to estimate the right-hand side of equation 2.5 (for $i=3$ ). The analysis showed that contributions to the right-hand side of equation 2.5 from the mean flow components (advection and dissipation) were in general small, and so in this case the mean dynamic pressure gradient can be reasonably approximated using equation 2.6. Measurements of $-\partial w^{2} / \partial x_{3} \approx \partial P / \partial x_{3}$ are shown in figure 6 . In figure 6 measurements of $\partial w^{2} / \partial x_{3}$ have been normalised using the integral length scale $\ell_{0}$ and the expected value of the rms of fluctuating velocity components at the edge of the boundary-affected region $u_{\delta, 0}$. Note that at the edge of the boundary-affected region $w / u \approx 1$ such that $u_{\delta, 0}$ and $w_{\delta, 0}$ are approximately equal and these values can be used interchangeably.

The data in figure 6 indicates that within the boundary-affected region $\partial w^{2} / \partial x_{3}$ increases in magnitude (and is negative in sign) as the boundary is approached, such that (according to equation 2.6) there is an increase in the mean dynamic pressure gradient. Consequently, as the fluid approaches the boundary it undergoes an increase in mean dynamic pressure at the expense of the TKE (see, for example, Hunt \& Graham 1978). Hunt \& Graham (1978) predicted that for otherwise spatially homogeneous and isotropic turbulence interacting with an impermeable boundary this effect leads to a reduction in TKE in the boundary-affected region. However, the data reported in section 3.2 show that the same effect does not hold here when the turbulence interacts with an effectively impermeable boundary (i.e. $R e_{K} \lesssim 0.2$ ), due to inhomogeneity in the $x_{3}$ direction. That is, the blocking effect of an impermeable boundary on the vertical energy flux results in additional energy contained within the boundary-affected region, relative to the same region in the absence of the boundary (McCorquodale \& Munro 2017). As a consequence, in the boundary-affected region we observe an increase in both TKE and mean dynamic pressure.

However, the data in figure 6 indicates that as $R e_{K}$ increases there is a reduction in the magnitude of $\partial w^{2} / \partial x_{3}$ and thus the degree by which the mean dynamic pressure gradient 


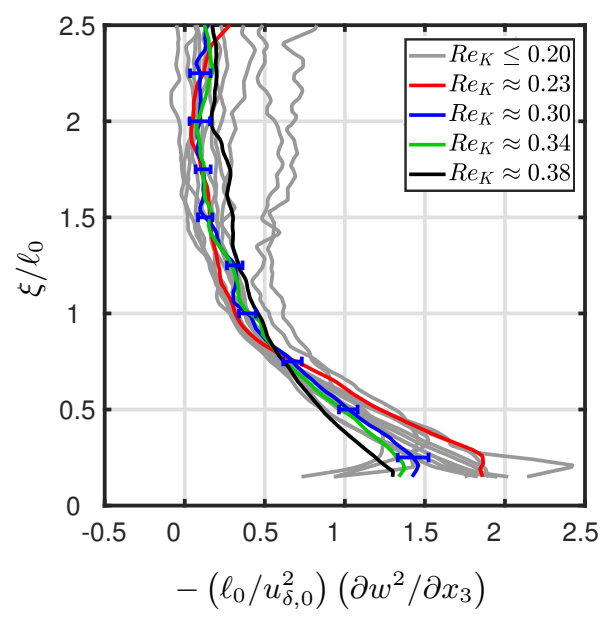

Figure 6. Measurements of $\partial w^{2} / \partial x_{3}$ plotted against scaled height $\xi / \ell_{0}$. A single data-set is shown for each experimental conditions reported in table 1, which is an average of the measurements obtained across the $n=5$ repeats conducted for each condition. The data at different $R e_{K}$ are shown separately, irrespective of $R e_{G}$. Also shown are representative error bars for the case $R e_{K} \approx 0.3$, corresponding to the standard error across the $n=5$ repeats.

increases within the boundary-affected region diminishes. The corresponding reduction in mean dynamic pressure (relative to an impermeable boundary) indicates there is a reduction in the blocking of turbulent motions by the boundary as $R e_{K}$ increases, consistent with the previous results. This interpretation of the velocity measurements has an important implication. If there is a reduction in blocking of turbulent motions by the boundary this must be as a result of transportation of turbulent energy into the boundary; in other words, there is a reduction in the formation of high-pressure stagnation events as fluid elements impinge onto the boundary (so-called "splats"). Crucially, it is thought that the imbalance between splats and antisplats (fluid elements ejecting away from the boundary as a result of high pressure collisions between fluid parcels) is central to intercomponent energy transfer. Consequently, a reduction in the formation of high pressure splats as $R e_{K}$ increases is thought to inhibit intercomponent energy transfer and also contribute to the reduction in the observed values of $\langle u\rangle_{1} / u_{0}$, shown in figure $3(\mathrm{~b})$, as $R e_{K}$ increases. This effect is investigated in more detail in section 4 .

\section{Analysis of intercomponent energy transfer}

The analysis in section 3 identified the flux of TKE as a prominent mechanism in determining the statistical structure of turbulence in the boundary-affected region. Recall, the transport of TKE by velocity and pressure fluctuations is described by the terms $T_{i j}$ and $\Pi_{i j}^{d}$ in the transport equations of the Reynolds stress tensor (i.e. equation 2.1) and the transport equation for TKE (i.e. equation 2.2). Here we investigate intercomponent energy transfer in order to derive additional insight into the governing dynamics. Intercomponent energy transfer is described by the pressure-strain correlation term $\Pi_{i j}^{s}$ of the transport equation of the Reynolds stress tensor (i.e. equation 2.1), which in the boundary-affected region is thought to describe an imbalance in the energy associated with splats and antisplats (see for example Perot \& Moin 1995; Bodart et al. 2010). In this context, splats and antisplats should not be confused with coherent structures that occur in a boundary layer subject to mean shear. Rather, splats are simply 
regions of fluid (or eddies) that move towards and impinge upon a surface whilst antisplats are regions of fluid that accelerate as they move away from the surface of a boundary (Perot \& Moin 1995). At an impermeable boundary, the genesis of antisplats has been directly linked to the process of splats impinging on a surface. That is, as a splat impinges upon an impermeable surface it slows down and may exhibit a tendancy to travel parallel to that surface. The subsequent collision of two fluid elements travelling parallel to the surface will give rise to an antisplat (Perot \& Moin 1995; Hunt \& Morrison 2000). In addition, as a vortical eddy approaches and interacts with an impermeable surface (i.e. a splat) it will generate regions of opposite vorticity; the interaction of these regions of opposite vorticity will in turn result in the ejection of fluid away from the surface (i.e. an antisplat) (Hunt \& Morrison 2000; Bodart et al. 2010; McCorquodale \& Munro 2018a). Different mechanisms of generating antisplats are expected at a permeable surface, which are discussed in section 4.2 .

Recently, McCorquodale \& Munro (2018a) analysed intercomponent energy transfer using a statistical approach, related to Quadrant-Hole Analysis (see, for example, Zhu et al. 2007, and references therein), to identify and isolate events within the turbulent flow that exhibit characteristics expected of splats and antisplats. That is, the stagnation flow associated with splats and antisplats is thought to exhibit strongly decelerating flow (with correspondingly large spatial gradients in $u_{3}^{\prime}$ ) and McCorquodale \& Munro (2018a) used this feature as a criterion to first identify splats and antisplats and then to evaluate conditional turbulent statistics associated with splats and antisplats. Here we have applied the same approach to the data obtained using the permeable boundaries.

\subsection{Criteria for isolating splats and antisplats}

It is instructive to revisit the criterion used by McCorquodale \& Munro (2018a) to identify splats and antisplats. Splat events moving towards the boundary $\left(u_{3}^{\prime}>0\right)$ yield negative values of the vertical strain rate $\partial u_{3}^{\prime} / \partial x_{3}$ and antisplat events moving away from the boundary $\left(u_{3}^{\prime}<0\right)$ yield positive values of the vertical strain rate $\partial u_{3}^{\prime} / \partial x_{3}$ (Perot \& Moin 1995; Bodart et al. 2010; Magnaudet 2003). However, fluid elements that exhibit these statistical characteristics exist throughout the entire flow, as a consequence of the random structure of a turbulent flow. On the other hand, the blocking associated with the formation of splats and antisplats is expected to give rise to more strongly decelerating flow than observed in the bulk interior of the flow. McCorquodale \& Munro (2018a) proposed that splats and antisplats may be isolated from the background flow by use of quadrant analysis on measurements of $u_{3}^{\prime}<0$ and $\partial u_{3}^{\prime} / \partial x_{3}$, and by further applying a threshold or critical value on the magnitude of the vertical strain rate $\partial u_{3}^{\prime} / \partial x_{3}$, defined in comparison to a reference value of strain rate given by $w / \bar{\ell}_{w}$ evaluated at the corresponding $x_{3}$. The reference strain rate was devised from an order of magnitude analysis (Bodart et al. 2010; McCorquodale \& Munro 2018a); when a packet of size $\ell$ meets an impermeable surface with velocity $u_{3}^{\prime}$, the vertical strain rate can be estimated as $u_{3}^{\prime} / \ell$. For a given point within the boundary-affected region, the average velocity and size of a fluid packet is given by $w$ and $\bar{\ell}_{w}$, where $\bar{\ell}_{w}$ denotes the time-averaged transverse integral length scale of the boundary-normal velocity component $u_{3}^{\prime}$, thus giving an average reference strain rate of $w / \bar{\ell}_{w}$.

By analysing the magnitude of vertical strain rate both in the boundary affected region and bulk interior of the flow, McCorquodale \& Munro (2018a) found the condition $\left|\left(\bar{\ell}_{w} / w\right) \partial u_{3}^{\prime} / \partial x_{3}\right|>6$ was sufficient to isolate splats and antisplats from background fluctuations. That is, the probability that the magnitude of the instantaneous vertical strain rate exceeds $6 w / \bar{\ell}_{w}$ was found to be negligible outside the boundary-effected region (see figure 7 ), and thus strain rates that exceed this value within the boundary-effected 
region were thought to correspond to fluid elements that exhibit strongly decelerating flow in response to the blocking effect of a surface.

To investigate how boundary permeability influences splat and antisplat formation we consider the probability of events that exhibit characteristics expected of splats and antisplats. That is, we split velocity measurements into 4 quadrants based upon the sign of $u_{3}^{\prime}$ and $\partial u_{3}^{\prime} / \partial x_{3}$ and calculate:

$$
\begin{gathered}
\text { Quadrant } 1\left(Q_{1}\right): \mathrm{P}_{Q_{1}} \equiv\left\langle\mathrm{P}\left(\frac{\bar{\ell}_{w}}{w} \frac{\partial u_{3}^{\prime}}{\partial x_{3}}>\phi_{T} \cap u_{3}^{\prime}>0\right)\right\rangle_{1}, \\
\text { Quadrant 2(Q }): \mathrm{P}_{Q_{2}} \equiv\left\langle\mathrm{P}\left(\frac{\bar{\ell}_{w}}{w} \frac{\partial u_{3}^{\prime}}{\partial x_{3}}>\phi_{T} \cap u_{3}^{\prime}<0\right)\right\rangle_{1}, \\
\text { Quadrant } 3\left(Q_{3}\right): \mathrm{P}_{Q_{3}} \equiv\left\langle\mathrm{P}\left(\frac{\bar{\ell}_{w}}{w} \frac{\partial u_{3}^{\prime}}{\partial x_{3}}<-\phi_{T} \cap u_{3}^{\prime}<0\right)\right\rangle_{1}, \\
\text { Quadrant } 4\left(Q_{4}\right): \mathrm{P}_{Q_{4}} \equiv\left\langle\mathrm{P}\left(\frac{\bar{\ell}_{w}}{w} \frac{\partial u_{3}^{\prime}}{\partial x_{3}}<-\phi_{T} \cap u_{3}^{\prime}>0\right)\right\rangle_{1},
\end{gathered}
$$

as a function of $\xi / \ell_{0}$, where $\mathrm{P}(A \cap B)$ denotes the probability of $A$ and $B$ and $\phi_{T}$ denotes a (positive) constant. When $\phi_{T}=0$ we consider the probabilities of each quadrant $P_{Q_{i}}$ based upon every measurement of the flow field (i.e. $P_{Q_{1}}+P_{Q_{2}}+P_{Q_{3}}+P_{Q_{4}}=1$ when $\left.\phi_{T}=0\right)$. When $\phi_{T}>0$ the evaluated probabilities associated with each quadrant decrease because those events that exhibit $\left|\left(\bar{\ell}_{w} / w\right) \partial u_{3}^{\prime} / \partial x_{3}\right|<\phi_{T}$ are excluded from the analysis. The value of $\phi_{T}$ used to isolate splats $\left(Q_{4}\right)$ and antisplats $\left(Q_{2}\right)$ from the background fluctuations, identified previously by McCorquodale \& Munro $(2018 a)$, is $\phi_{T}=6$. In the following, a comparison of the probabilities $P_{Q_{2}}$ and $P_{Q_{4}}$ when $\phi_{T}=0$ and $\phi_{T}=6$ enables us to comment on how boundary permeability effects the magnitude of the strain rates as fluid elements impinge onto the surface of a porous media.

We note that when evaluating the statistics described by equation 4.1 anomalous trends were identified for experiments at $R e_{G} \approx 2020$ and 4220 when $\xi / \ell_{0} \lesssim 0.25$. We attribute this effect to a data processing issue relating to the computation of the strain rates (McCorquodale \& Munro 2018a). That is, within the viscous sublayer, the fluid velocities are much smaller than within the bulk of the flow, such that the uncertainty within these measurements increases as a proportion of the fluid velocity. Thus, the error associated with these measurements may give rise to unreliable estimates of the strain rate. This effect is thought to be most prevalent at low Reynolds number as a result of the lower fluid velocities that occur in these experiments. Consequently, measurements of $P_{Q_{i}}$ are not reported here for $\xi / \ell_{0} \lesssim 0.25$ when $R e_{G} \approx 2020$ and 4220 . We also note that, since we were unable to fully resolve the dissipative scales within the flow (see section 2.2), this may lead to a slight underestimate of the instantaneous vertical strain $\partial u_{3}^{\prime} / \partial x_{3}$ in the experiment data. However, we stress that in this section we are primarily concerned with fluid elements as they are blocked by the boundary - a process which primarily influences the large scales within the flow (McCorquodale \& Munro 2018a). Therefore, the small scales are not thought to be dynamically significant within this analysis; the small unresolved scales are not blocked by the surface except within $\xi / \ell_{0} \lesssim 0.1$.

Measurements of $P_{Q_{i}}$, evaluated when $\phi_{T}=0$ and 6 , are shown in figure 7 . Figure 7 shows that there is an increase in the overall probability (given by $\phi_{T}=0$ ) of $P_{Q_{2}}$ and $P_{Q_{4}}$ within the boundary-affected region $\left(\xi / \ell_{0}<1\right)$, whilst there is a corresponding reduction in the overall probability of $P_{Q_{1}}$ and $P_{Q_{3}}$. When $\phi_{T}=0$ there is little, if any, evident dependence on $\operatorname{Re}_{K}$. Figure 7 also shows $P_{Q_{1}}$ and $P_{Q_{3}}$ are negligible in the boundary- 

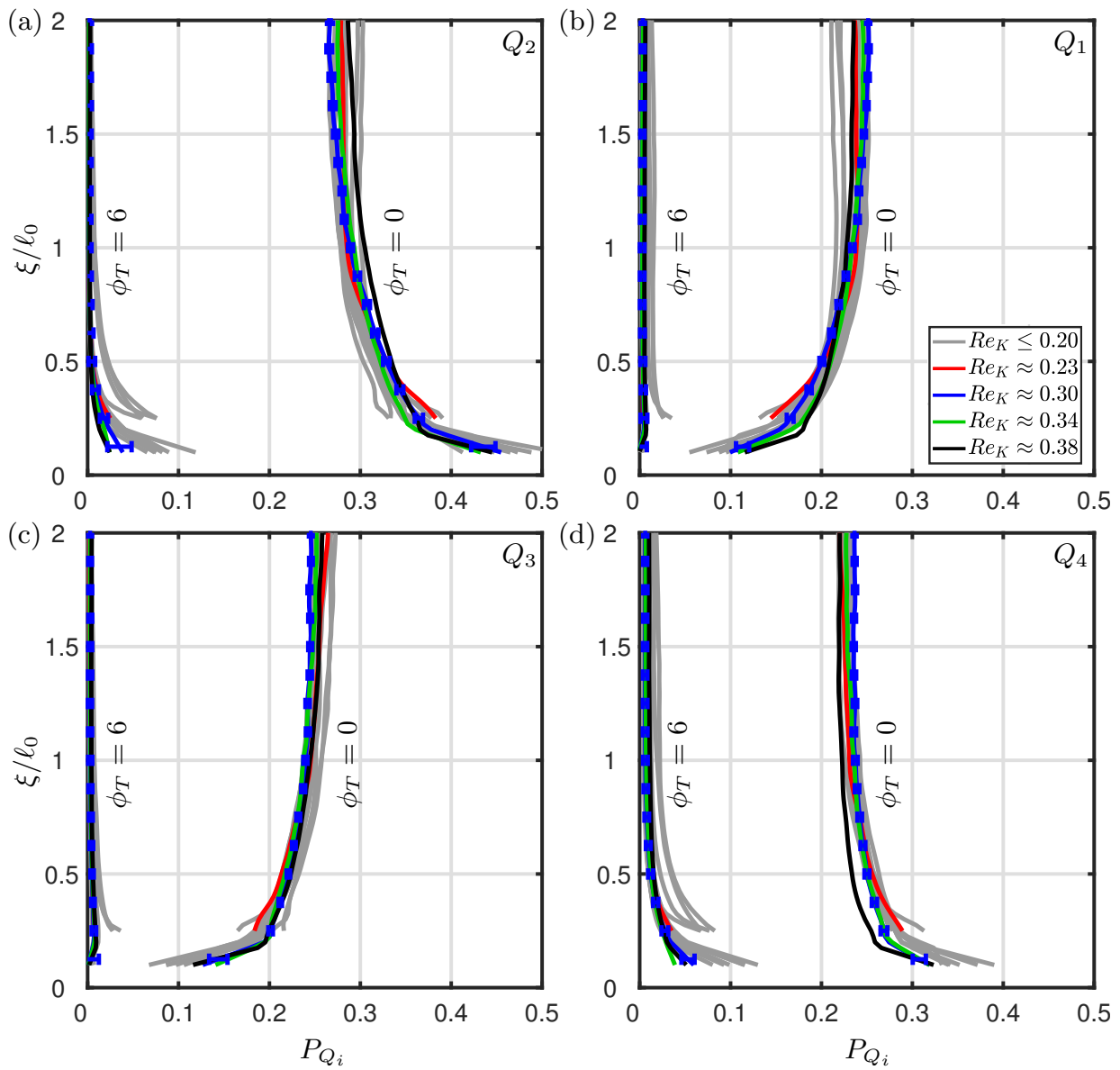

FiguRE 7 . Measurements of the probability that the normalised strain rate $\left(\bar{\ell}_{w} / w\right) \partial u_{3}^{\prime} / \partial x_{3}$ surpasses a given constant $\phi_{T}$ (the values of $\phi_{T}$ are shown in each plot). Data are separated into quadrants according to the sign of $u_{3}^{\prime}$ and $\partial u_{3}^{\prime} / \partial x_{3}$, as described by equation (4.1); (a) $Q_{2}$, (b) $Q_{1}$, (c) $Q_{3}$ and (d) $Q_{4}$. In each plot a single data-set is shown for each experimental condition reported in table 1 , which is an average of the measurements obtained across the $n=5$ repeats conducted for each condition. The data at different $R e_{K}$ are shown separately, irrespective of $R e_{G}$. Also shown are representative error bars for the case $R e_{K} \approx 0.3$, corresponding to the standard error across the $n=5$ repeats.

affected region when $\phi_{T}=6$. In contrast, the probabilities $P_{Q_{2}}$ and $P_{Q_{4}}$ evaluated when $\phi_{T}=6$ increase within the boundary-affected region, although the magnitude of the increase in $P_{Q_{2}}$ and $P_{Q_{4}}$ reduces as $R e_{K}$ increases, as shown in figure 7 .

For an effectively impermeable boundary $\left(R e_{K} \lesssim 0.2\right)$, figure 7 indicates that the increase in probability of $P_{Q_{2}}$ and $P_{Q_{4}}$ when $\phi_{T}=6$ is approximately equal to the increase in probability when $\phi_{T}=0$. Thus, the increase in $P_{Q_{2}}$ and $P_{Q_{4}}$ is primarily driven by events that satisfy the condition $\phi_{T}=6$, which we interpret as a signature of splats and antisplats due to the inhibiting effects of the boundaries on the turbulence (McCorquodale \& Munro 2018a).

However, for a permeable boundary $\left(R e_{K} \gtrsim 0.2\right)$, figure $7(\mathrm{a}, \mathrm{d})$ shows that the increase in probability of $P_{Q_{2}}$ and $P_{Q_{4}}$ when $\phi_{T}=6$ is smaller than the corresponding increase in probability when $\phi_{T}=0$. Thus the increase in $P_{Q_{2}}$ and $P_{Q_{4}}$ is driven by events which do not satisfy the condition $\phi_{T}=6$ (i.e. which exhibit $\left|\left(\bar{\ell}_{w} / w\right) \partial u_{3}^{\prime} / \partial x_{3}\right|<6$ ). That is, 
the increase in $P_{Q_{2}}$ and $P_{Q_{4}}$ when $\phi_{T}=0$ indicates that fluid elements impinging onto a permeable boundary are still inhibited by the presence of the boundary at the same frequency as if the boundary were impermeable, but the magnitude of the strain rates associated with these interactions are smaller than occur at an impermeable boundary. This effect can be attributed to a reduction in the inhibiting effects of the permeable boundary as $R e_{K}$ increases, consistent with the results of section 3. Crucially, these results indicate that as $R e_{K}$ increases there is a reduction in events exhibiting strongly decelerating flow (i.e. splats and antisplats) in which intercomponent energy transfer is thought to be concentrated (see, for example, Perot \& Moin 1995; Bodart et al. 2010).

\subsection{Conditional statistics}

In this section we seek to consider explicitly how boundary permeability influences intercomponent energy transfer by computing conditional turbulent statistics from measurements of the flow that exhibit characteristics expected of splats and antisplats. We adopt the approach of McCorquodale \& Munro (2018a) and compute conditional rms values of $u_{1}^{\prime}$ where the criteria described in section 4.1 to isolate splats and antisplats are used as conditioning events. That is, intercomponent energy transfer is thought to be governed by an imbalance in the energy associated with splats and antisplats (see, for example Perot \& Moin 1995; Bodart et al. 2010) and here we investigate how this imbalance depends on $R e_{K}$. We denote the rms values of $u_{1}^{\prime}$ associated with measurements of the flow indicative of splats and antisplats by $u_{Q_{4}}$ and $u_{Q_{2}}$ respectively. Hence, $u_{Q_{2}}$ and $u_{Q_{4}}$ are rms values of $u_{1}^{\prime}$ given that the measurements used to compute these statistics satisfy the conditions $\left(\bar{\ell}_{w} / w\right) \partial u_{3}^{\prime} / \partial x_{3}>\phi_{T} \cap u_{3}^{\prime}<0$ and $\left(\bar{\ell}_{w} / w\right) \partial u_{3}^{\prime} / \partial x_{3}<-\phi_{T} \cap u_{3}^{\prime}>0$, respectively. Values of $u_{Q_{4}}$ and $u_{Q_{2}}$ have been computed for both $\phi_{T}=0$ and $\phi_{T}=6$. That is, conditional $u_{Q_{2}}$ and $u_{Q_{4}}$ statistics associated with specifically splats and antisplats have been computed using the critical value $\phi_{T}=6$ (McCorquodale \& Munro 2018a) and the results compared against conditional statistics evaluated for $\phi_{T}=0$. This comparison enables us to illustrate how the effects of splats and antisplats on the average statistical structure of the flow are influenced by $R e_{K}$. To ensure the use of robust statistics, measurements of $u_{Q_{2}}\left(\phi_{T}=6\right)$ and $u_{Q_{4}}\left(\phi_{T}=6\right)$ are only reported for $\xi / \ell_{0} \leqslant 0.6$ since the probability that measurements of the flow exhibit $\left|\left(\bar{\ell}_{w} / w\right) \partial u_{3}^{\prime} / \partial x_{3}\right|>6$ (i.e. strongly decelerating flow indicative of splats and antisplats) is negligible for $\xi / \ell_{0} \gtrsim 0.6$ (see section 4.1). In addition, measurements of $u_{Q_{2}}$ and $u_{Q_{4}}$ are only reported for $\xi / \ell_{0} \gtrsim 0.25$ when $R e_{G} \approx 2020$ and 4220 for the reasons explained in section 4.1 .

Measurements of $u_{Q_{2}}$ and $u_{Q_{4}}$ are shown in figure 8 . We note that $u_{Q_{4}}\left(\phi_{T}=0\right)>$ $u_{Q_{2}}\left(\phi_{T}=0\right)$ outside the boundary-affected region (i.e. for $\left.\xi / \ell_{0}>1\right)$, which indicates that the energy associated with turbulent motions incident towards the boundary exceeds that of turbulent motions moving away from the boundary (recall that $u_{Q_{4}}$ satisfy the condition $u_{3}^{\prime}>0$ and $u_{Q_{2}}$ the condition $\left.u_{3}^{\prime}<0\right)$. We stress that this is simply a result of the anisotropic nature of the flow produced by OGT (see section 3.2).

Within the boundary-affected region (i.e. for $\left.\xi / \ell_{0}<1\right)$ permeability effects are apparent. At an effectively impermeable boundary $\left(R e_{K} \lesssim 0.2\right)$ results are consistent with previous investigations studying the interaction of OGT with an impermeable boundary (McCorquodale \& Munro 2018a). Figure 8(a) shows that $u_{Q_{4}}\left(\phi_{T}=0\right) / u_{Q_{2}}\left(\phi_{T}=0\right)$ reduces at the edge of the boundary-affected region $\left(\xi / \ell_{0} \approx 0.6\right.$ to 1.0$)$ and approaches a value of approximately 1 . This trend physically represents a change in the imbalance between the energy associated with turbulent motions incident towards the boundary, which are increasingly less energetic, relative to the energy of turbulent motions moving away from the boundary. McCorquodale \& Munro (2018a) attributed this result to 

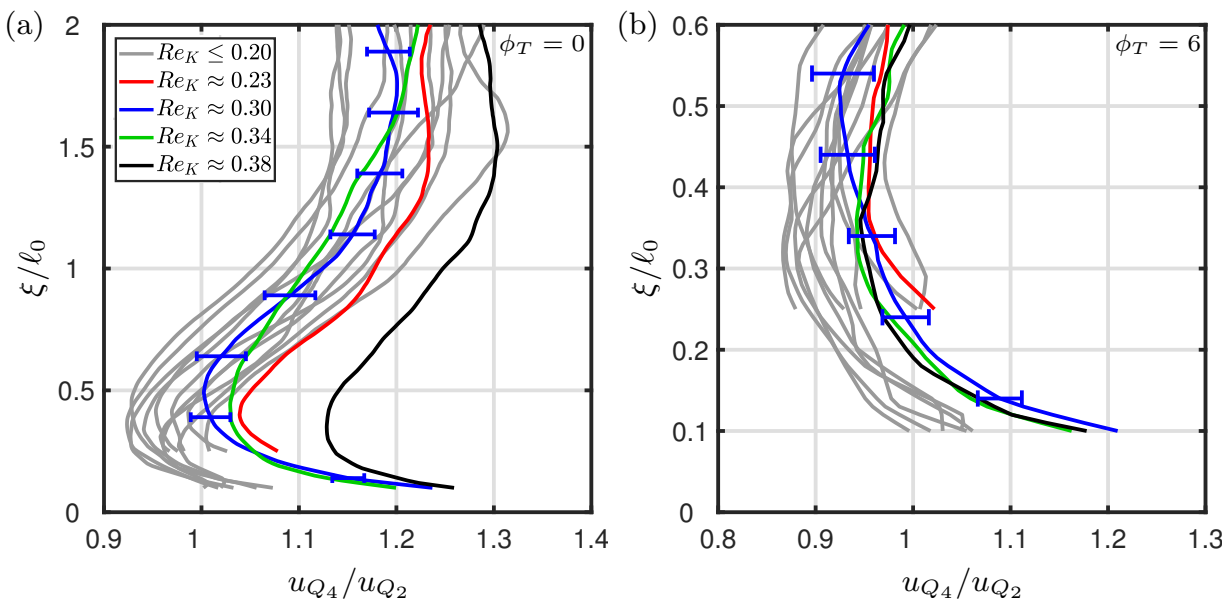

FIGURE 8. (a) RMS measurements $u_{Q_{2}}$ and $u_{Q_{4}}$ of the conditionally sampled horizontal velocity component plotted against scaled height above the boundary $\xi / \ell_{0}$ subject to the condition $\phi_{T}=0$. The data has been normalised to allow a direct comparison between the magnitude of $u_{Q_{2}}$ and $u_{Q_{4}}$. (b) Shows the same data as (a) subject to the condition $\phi_{T}=6$. In each plot a single data-set is shown for each experimental condition reported in table 1, which is an average of the measurements obtained across the $n=5$ repeats conducted for each condition. The data at different $R e_{K}$ are shown separately, irrespective of $R e_{G}$ [see legend in (a) which applies to all plots]. Also shown are representative error bars for the case $R e_{K} \approx 0.3$, corresponding to the standard error across the $n=5$ repeats.

the effects of turbulent transport, due to the blocking effect of the boundary on the boundary-normal TKE flux (see section 3.2), which in isolation would act to result in $u_{Q_{4}} \approx u_{Q_{2}}$. An imbalance in $u_{Q_{2}}$ and $u_{Q_{4}}$ is re-established as the boundary is further approached; for the region $0.3 \lesssim \xi / \ell_{0} \lesssim 0.6$ we note that $u_{Q_{2}}\left(\phi_{T}=0\right)>u_{Q_{4}}\left(\phi_{T}=0\right)$ and $u_{Q_{2}}\left(\phi_{T}=6\right)>u_{Q_{4}}\left(\phi_{T}=6\right)$, whilst the ratio $u_{Q_{4}} / u_{Q_{2}}$ continues to decrease. Hence, over this finite region the energy associated with turbulent motions moving away from the boundary exceeds that of the energy of turbulent motions incident towards the boundary. McCorquodale \& Munro (2018a) concluded that this effect arose as a result of a net intercomponent energy transfer from $w^{2}$ to $u^{2}$ associated with splats and antisplats. Viscous effects dominate closer to the boundary and so $u_{Q_{4}} / u_{Q_{2}}$ increases within the viscous sublayer $\left(\xi / \ell_{0} \lesssim 0.3\right)$ as the imbalance betweens splats and antisplats is governed by the dissipation of TKE (Perot \& Moin 1995).

Figure $8\left(\right.$ a) shows similar trends are observed at a permeable boundary $\left(R e_{K} \gtrsim 0.2\right)$; $u_{Q_{4}} / u_{Q_{2}}$ also reduces over the region $0.3 \lesssim \xi / \ell_{0} \leqslant 1$ and increases over the region $\xi / \ell_{0} \lesssim 0.3$. However, figure $8(\mathrm{a})$ also shows that within the boundary-affected region the magnitude of $u_{Q_{4}} / u_{Q_{2}}$ increases as $R e_{K}$ increases, such that $u_{Q_{4}}\left(\phi_{T}=0\right)>u_{Q_{2}}\left(\phi_{T}=\right.$ $0)$ throughout the boundary-affected region. This trend can be explained, in part, by effects associated with turbulent transport; as $R e_{K}$ increases there is a reduction in the kinematic blocking effect of the boundary on the TKE flux (see figure 4) such that, on average, turbulent motions incident towards the boundary are more energetic than turbulent motions moving away from the boundary (see figure 4). That is, when fluid penetrates into the porous media, as the kinematic blocking effect of the boundary is inhibited for increasing $R e_{K}$, an equivalent volume of fluid is expelled from the porous media. However, fluid expelled from the porous media is (on average) less energetic than the fluid that penetrates the porous media as a result of the high viscous dissipation that occurs within the porous media (see below for details). 
However, we can deduce insight into intercomponent energy transfer by contrasting the results shown in figure 8(a) against those shown in figure $8(\mathrm{~b})$, in which we have isolated strongly decelerating events in which intercomponent energy transfer is thought to be concentrated (see, for example Perot \& Moin 1995; Bodart et al. 2010). That is, figure $8(\mathrm{~b})$ shows that $u_{Q_{2}}>u_{Q_{4}}$ over $0.3 \lesssim \xi / \ell_{0} \lesssim 0.6$ when $\phi_{T}=6$, even as $R e_{K}$ increases, consistent with results from effectively impermeable boundaries $\left(R e_{K} \lesssim 0.2\right)$. This result implies that at a permeable boundary $\left(R e_{K} \gtrsim 0.2\right)$ strongly decelerating flow within the boundary layer (i.e. splats and antisplats) is associated with an intercomponent energy transfer from $w^{2}$ to $u^{2}$, as observed at impermeable boundaries. However, we stress that, on average, turbulent motions incident towards a permeable boundary exhibit smaller values of the vertical strain rate $\partial u_{3}^{\prime} / \partial x_{3}$ than equivalent motions at an impermeable boundary, since turbulence is able to penetrate the boundary. Consequently as $R e_{K}$ increases only a reducing proportion of fluid elements form splats and antisplats (see figure 7). Therefore, at permeable boundaries there is an overall reduction in intercomponent energy transfer over this region, due to the depletion of splats and antisplats in the boundary effected region. Consequently, we attribute the increase in $u_{Q_{4}}\left(\phi_{T}=0\right) / u_{Q_{2}}\left(\phi_{T}=0\right)$ for permeable boundaries, shown in figure 8(a), to the combined effects of a reduction in the turbulent transport and a reduction in intercomponent energy transfer as $R e_{K}$ increases.

A limitation in our analysis is that it does not identify the precise changes in dynamics associated with splat and antisplat formation that occur as boundary permeability increases. We note that Perot \& Moin (1995) proposed a model describing splat and antisplat formation above a permeable boundary; they argue that splats and antisplats still exist above a permeable boundary, but that they no longer represent stagnation-point regions of the flow. Instead, Perot \& Moin (1995) suggest that splats and antisplats above a permeable boundary represent regions of fluid about to pass through the boundary; as the fluid elements pass through the boundary they distort and transfer energy between the velocity components. However, viscous effects close to the boundary dissipate energy from the fluid elements such that on-average antisplats, which emerge from a region of large viscous dissipation, are less energetic than splats. As a result there is an intercomponent energy transfer from splats to antisplats that is governed by the viscous dissipation. This dissipative mechanism is similar to that which Perot \& Moin (1995) proposed governed intercomponent energy transfer at a solid impermeable boundary. We stress that results for the permeable boundaries $\left(R e_{K} \gtrsim 0.2\right)$ shown in figure 8 are consistent with this model for $\xi / \ell_{0} \lesssim 0.3$, over which significant viscous dissipation occurs (see figure 4).

Outside this dissipative region, McCorquodale \& Munro (2018a) and Bodart et al. (2010) argued that the net imbalance between splats and antisplats at an impermeable boundary arises in part from the formation of antisplats through so-called self-generation mechanisms (Bodart et al. 2010; Hunt \& Morrison 2000). That is, recall that antisplats can form due to the interactions of two regions of opposite vorticity when a splat approaches a boundary; as a splat is blocked by the boundary, the no-slip condition results in instantaneous shear and the vorticity associated with this shear acts to eject fluid away from the boundary. The more complicated dynamics arising in this interaction were not considered in the model of Perot \& Moin (1995), in which splats and antisplats arise as simple consequences of the equation of continuity. McCorquodale \& Munro $(2018 a)$ argued that this mechanism gives rise to a net energy transfer from $w^{2}$ to $u^{2}$ outside the viscous sublayer. We note that for $R e_{K} \lesssim 0.2$ results shown in figure 8 over $0.3 \lesssim \xi / \ell_{0} \lesssim 0.6$ are consistent with an imbalance in energy of splats and antisplats that 
results from a net energy transfer from $w^{2}$ to $u^{2}$, in accordance with this mechanism. It is logical then to consider how this mechanism may be influenced by boundary permeability.

Inferences into how the self-generation mechanism of antisplat formation may be influenced by boundary permeability can be made from studies investigating the interaction of vortex rings with boundaries. That is, studies investigating the interaction of a vortex ring with solid impermeable boundaries report a rebound of the vortex ring away from the boundary (see for example Walker et al. 1987; Orlandi \& Verzicco 1993; Munro et al. 2009; Munro 2012) which appears similar, in concept, to the antisplat self-generation mechanism. However, studies investigating the interaction of vortex rings with permeable boundaries indicate that vortex ring rebound is inhibited as boundary permeability increases (Mujal-Colilles et al. 2015); the relaxation of the kinematic blocking condition facilitates penetration of the flow into the boundary and a reduction in instantaneous shear such that the vorticity that acts to eject fluid away from the boundary is weaker. The results presented here are consistent with these observations; we have observed a relaxation of the kinematic blocking effect of the boundary (section 3) that reduces the strain rate of fluid elements as they impinge onto the boundary (section 4.1). Consequently, we propose that the "self-generation" mechanism of antisplat formation is inhibited as $R e_{K}$ increases, thereby depleting the formation and splats and antisplats and contributing to the observed reduction in intercomponent energy transfer.

\section{Discussion}

In this section we focus on the interpretation of our results in the wider context of the interaction of turbulent flows with permeable boundaries. We note that in this study we have considered only a limited range of $R e_{K}$; recall that this description of a permeable media characterises the behaviour at the boundary as intermediate between the limits of a solid impermeable boundary and unconfined flow. The data reported in sections 3 and 4 illustrate that the insight derived here is applicable to permeable media in which the boundary still exerts a significant influence on the flow. Nonetheless, it is anticipated that the results obtained here can be widely applied for two reasons. Firstly, the range of $R e_{K}$ considered here is applicable to a number of examples of turbulent flows interacting with boundaries. For example, sediments beds in rivers and marine systems typically exist in the range $R e_{K} \sim 10^{-3}$ to 10 (Voermans et al. 2017). [We note that Voermans et al. (2017) adopt the definition of $R e_{K}$ given by equation 2.7, which is different from that used in this study (equation 2.9). However, the underlying interpretation of $R e_{K}$ is identical in both cases (see section 2.4) and consequently we anticipate the values of $R e_{K}$ for sediment beds to be of the same order of magnitude when using either algebraic definition of $R e_{K}$.] Secondly, the results reported provide insight into the underlying physical mechanisms that govern the interaction with boundaries over the important transition between impermeable and permeable regimes. However, a limitation of this study is that we have only considered porous media of a single value of porosity (97\%). Thus, further study using porous media of lower porosities is required to establish to what extent the current results depend on the structure of the porous media used.

The data reported in sections 3 and 4 show that increasing the permeability Reynolds number results in a gradual reduction in the inhibiting effects of the boundary through a relaxation of the macroscopic blocking condition. This gives rise to a reduction in the blocking of the vertical flux of TKE and a suppression of intercomponent energy transfer. Recall, these physical phenomena are described by the turbulent transport and pressure strain terms of equations 2.1 and 2.2 (see section 2.3). We stress that the results obtained here using permeable boundaries are distinctly different from those 
reported by Perot \& Moin (1995), in which the interaction of zero-mean-shear turbulence and a perfectly permeable boundary was studied through Direct Numerical Simulation. These differences are due to the fundamentally different boundary conditions used; by studying the perfectly permeable boundary, Perot \& Moin (1995) sought to remove the blocking effect of the boundary entirely and isolate near-boundary viscous effects within the interaction. Consequently, the governing mechanisms that are prevalent in their study are simpler than those observed here. This comparison illustrates the sensitivity of the interaction between turbulence and a permeable boundary to the boundary conditions imposed on the boundary normal velocity component. That is, numerical studies can only accurately reproduce the dynamics and statistical structure of turbulence in the interface region with proper selection of boundary conditions imposed by a permeable media.

These results emphasise the care that must be taken when adopting the use of a model, such as the "slip velocity" model (see section 1), to describe the boundary conditions applied at the boundary of a porous media. We note that the original slip velocity boundary conditions proposed by Beavers \& Joseph (1967) was developed for use in laminar flow, but this model has subsequently been extended to turbulent conditions (Hanh et al. 2002). Of particular note is the boundary condition utilised for the boundary-normal velocity component; these models enforce a no-penetration condition on the boundary-normal velocity component (i.e. $u_{3}^{\prime}=0$ ), which retains the kinematic blocking effect of the boundary on the boundary-normal velocity component as if the boundary were impermeable. In other words, the model proposed by Hanh et al. (2002) assumes that flow in permeable media is laminar and governed by Darcy's law. In essence, this assumes that the direct effects of boundary permeability on the turbulent velocity components, which have been isolated in this study, are small in the context of indirect effects associated with changes in mean shear within the interface region. We stress that the accuracy of this assumption will vary according to the flow under consideration. This approach should yield reliable results when $R e_{K} \ll 1$, since a permeable boundary will act as if it were impermeable to turbulent fluctuations and approximately enforce the no-penetration condition on the boundary-normal velocity component (i.e. $u_{3}^{\prime}=0$ ). However, results reported in the literature indicate that this assumption becomes unreliable in channel flows with permeable boundaries when $R e_{K} \gtrsim 1$; significant differences in both the statistical structure and dynamics of the interface region have been reported between studies using the slip-velocity model and those modelling flow inside the interface region as a continuum through the use of volume-averaged Navier-Stokes formulations (see, for example, Hanh et al. 2002; Breugem et al. 2006; Rosti et al. 2015). We attribute these differences to the significance of the blocking effect of permeable boundaries on turbulence fluctuations that is identified here. In short, the boundary conditions specified by the slip velocity model at the boundary of a permeable media may be insufficient to describe the complex interaction of the blocking effect of permeable boundaries on turbulent fluctuations in highly permeable media.

However, other factors will also influence the validity of the boundary conditions used within the slip velocity model proposed by Hanh et al. (2002). For example, so far we have only considered turbulent flow in which the depth of penetration of turbulent fluctuations into the permeable media is governed $R e_{K}$. However, in some applications the permeable media are sufficiently thin that the penetration depth is instead limited by an underlying impermeable surface, which has a profound influence on the dynamics and statistical structure of the flow (see, for example Li et al. 2020; Sharma \& García-Mayoral 2020). In this case, although increasing the permeability Reynolds numbers results in a relaxation of the macroscopic blocking condition at the surface of the permeable media, 
the boundary-normal velocity component is still inhibited in the interace region by the underlying surface at which the no-penetration condition is enforced (see, for example Li et al. 2020; Sharma \& García-Mayoral 2020). Consequently, in a thin permeable media the model boundary conditions proposed by Hanh et al. (2002) more closely approximates the boundary conditions applied at the boundary. In these cases it is conceivable that the slip-velocity condition may yield accurate results in turbulent channel flows even when $R e_{K} \gtrsim 1$. The direct validation of this hypothesis is an avenue for future study.

\section{Conclusion}

The interaction between oscillating-grid turbulence and solid and permeable boundaries (aligned parallel to the grid) has been studied experimentally. A permeability Reynolds number, $R e_{K}$, suitable for use in zero-mean-shear turbulence, is used to determine conditions under which boundary permeability effects become significant. The results indicate that when $R e_{K} \lesssim 0.2$ the boundary acts as if it were impermeable. In this case the interaction is dominated by the blocking of a far-field TKE flux by the kinematic blocking condition (McCorquodale \& Munro 2017), with secondary mechanisms acting through intercomponent energy transfers (Perot \& Moin 1995; Walker et al. 1996; McCorquodale \& Munro 2018a).

For $R e_{K} \gtrsim 0.2$ the inhibiting effects of the boundary are reduced, which results in both an increase in the magnitude of the boundary-normal rms turbulent velocity components and a reduction in the magnitude of the boundary-tangential rms turbulent velocity components. This is primarily attributed to a relaxation of the macroscopic blocking condition as turbulent motions penetrate into the porous media, such that there is a reduction in the blocking of the vertical flux of TKE. Intercomponent energy transfer is also found to be suppressed due to the macroscopic relaxation of the blocking condition. That is, the penetration of turbulence into the porous media inhibits the formation of rapidly-decelerating flow (splats) in the boundary-affected region; at an impermeable boundary the stagnation-flow associated with splat events gives rise to energy transfer between velocity components, but the relative occurrence of these events diminishes as turbulent motions penetrate the porous media such that energy transfer reduces overall. Within the viscous sublayer, dissipative effects that are dominant at an impermeable boundary (i.e. $R e_{K} \lesssim 0.2$ ) are also found to be prevalent even as $R e_{K}$ increases; we attribute this trend to the moderate $R e_{K}$ range used.

This study has enabled, for the first time, the direct effects of boundary permeability on turbulent fluctuations to be isolated from indirect effects, which are associated with changes in the mean shear. These results provide insight into the mechanisms that govern the interaction of turbulence with permeable boundaries in flows in which the mean shear is small, such that the dynamics of the interaction are governed by eddy impingement. Moreover, these results illustrate that turbulent structure over a permeable boundary is strongly influenced by the blocking effect of the boundary on the boundary-normal velocity component. In light of these observations, we argue that further analysis of the "slip velocity" model (Hanh et al. 2002), which does not consider this important aspect of the interaction, is required to establish conditions under which the model can be accurately utilised.

The authors acknowledge Jim Meakin and Steve Lawton for technical support with the experimental apparatus. MWM acknowledges financial support through an Engineering and Physical Sciences Research Council Studentship (number 1510466). 
Declaration of interests. The authors report no conflict of interest.

\section{REFERENCES}

Aronson, D., Johansson, A. V. \& LÖfdAhl, L. 1997 Shear-free turbulence near a wall. J. Fluid Mech. 338, 363-395.

Atkinson, J. F., Damiani, L. \& Harleman, D. R. F. 1987 Comparison of velocity measurements using a laser anemometer and a hot-film probe, with application to gridstirring entrainment experiments. Phys. Fluids 30, 3290-3292.

Bear, J. 2013 Dynamics of Fluids in Porous Media. Newburyport : Dover Publications.

Beavers, G. S. \& Joseph, D. D. 1967 Boundary conditions at a naturally permeable wall. J. Fluid Mech. 30(1), 197-207.

Bodart, J., Cazalbou, J. B. \& Joly, L. 2010 Direct numerical simulation of unsheared turbulence diffusing towards a free-slip or no-slip surface. J. Turbul. 11(48), 1-18.

Bottaro, A. 2019 Flow over natural or engineered surfaces: an adjoint homogenization perspective. Journal of Fluid Mechanics 877, P1.

Breugem, W. P., Boersma, B. J. \& Uittenbogand, R. E. 2006 The influence of wall permeability on turbulent channel flow. J. Fluid Mech. 562, 35-72.

Brinkman, H. C. 1947 A calculation of the viscous force exerted by a flowing fluid on a dense swarm of particles. Appl. Sci. Res. A 1, 27-34.

British Standards Institution 2010 BS EN ISO 11058:2010: Geotextiles and geotextilerelated products - Determination of water permeability characteristics normal to the plane, without load. London, England: BSI.

Brumley, B. H. \& JiRKA, G. H. 1987 Near-surface turbulence in a grid-stirred tank. J. Fluid Mech. 183, 235-263.

Cant, R., Castro, I. \& Walklate, P. 2002 Plane jets impinging on porous walls. Exp. Fluids 32, $16-26$.

Connolly, J.P., Armstrong, N.E. \& Miksad, R.W. 1983 Adsorption of hydrophobic pollutants in estuaries. J. Environ. Engng. 109, 17-35.

De Silva, I. P. D. \& Fernando, H. J. S. 1994 Oscillating grids as a source of nearly isotropic turbulence. Phys. Fluids 6, 2455-2464.

Defonseka, C. 2019 Water-Blown Cellular Polymers, A Practical Guide, 2nd edn. De Gruyter.

Efstathiou, C. \& Luhar, M. 2018 Mean turbulence statistics in boundary layers over highporosity foams. J. Fluid Mech. 841, 351-379.

Fernando, H. J. S. \& De Silva, I. P. D. 1993 Note on secondary flows in oscillating grid, mixing box experiments. Phys. Fluids 5, 1849-1851.

Hanh, S., Je, J. \& ChOI, H. 2002 Direct numerical simulation of turbulent channel flow with permeable walls. J. Fluid Mech. 450, 259-285.

Hannoun, I. A., Fernando, H. J. S. \& List, E. J. 1988 Turbulence structure near a sharp density interface. J. Fluid Mech. 189, 189-209.

Hassanizadeh, S. M. \& GRey, W. G. 1979 General conservation equations for multi-phase systems: 1. averaging procedure. Advances in Water Resources 2, 131-144.

Hassanizadeh, S. M. \& Grey, W. G. 1990 Mechanics and thermodynamics of multiphase flow in porous media including interphase boundaries. Advances in Water Resources 13, 169-186.

Hopfinger, B. J. \& Linden, P. F. 1982 Formation of thermoclines in zero-mean-shear turbulence subjected to a stabilizing buoyancy flux. J. Fluid Mech. 11, 157-173.

Hopfinger, B. J. \& Toly, J. A. 1976 Spatially decaying turbulence and its relation to mixing across density interfaces. J. Fluid Mech. 78(1), 155-175.

Hunt, J. C. R. 1984 Turbulence structure in thermal convection and shear-free boundary layers. J. Fluid Mech. 138), 161-184.

Hunt, J. C. R. \& Graham, J. M. R. 1978 Free-stream turbulence near plane boundaries. J. Fluid Mech. 84(2), 209-235.

Hunt, J. C. R. \& Morrison, J. F. 2000 Eddy structure in turbulent boundary layers. Eur. J. Mech. B - Fluids 19, 679-694. 
Johnson, B. A. \& Cowen, E. A. 2018 Turbulent boundary layers absent mean shear. J. Fluid Mech. 835, 217-251.

Katz, A. J. \& Thompson, A. H 1986 Quantitative prediction of permeability in porous rock. Phys Rev B 34(11), 8179-8181.

Keane, R. D. \& Adrian, R. J. 1990 Optimization of particle velocimeters. Part I: Double pulsed systems. Meas. Sci. Technol. 1, 1202-1215.

Kim, T, Blois, G., Best, J. L. \& Christensen, K. T. 2020 Experimental evidence of amplitude modulation in permeable-wall turbulence. J. Fluid Mech. 887, A3.

Kit, E. L. G., Strang, E. J. \& Fernando, H. J. S. 1997 Measurement of turbulence near shear-free density interfaces. J. Fluid Mech. 334, 293-314.

Kuwata, Y. \& SugA, K. 2016 Transport mechanism of interface turbulence over porous and rough walls. Flow Turbul. Combust 97, 1071-1093.

KuwAtA, Y. \& SugA, K. 2017 Direct numerical simulation of turbulence over anisotropic porous media. J. Fluid Mech. 831, 41-71.

LAGE, J. L. 1998 The fundamental theory of flow through permeable media from Darcy to turbulence. In Transport Phenomena in Porous Media (ed. D. B. Ingham \& I. Pop), chap. 1, pp. 1-30. Oxford: Elsevier Ltd.

Li, Q., Pan, M., Zhou, Q. \& Dong, Y. 2020 Turbulent drag modification in open channel flow over an anisotropic porous wall. Phys. Fluids 32, 015117.

Magnaudet, J. 2003 High-Reynolds-number turbulence in a shear-free boundary layer: revisiting the Hunt-Graham theory. J. Fluid Mech. 484, 167-196.

Manes, C., Poggi, D. \& Ridolfi, L. 2011 Turbulent boundary layers over permeable walls: scaling and near-wall structure. J. Fluid Mech. 687, 141-170.

Manes, C., Pokrajac, D., McEwan, I \& Nikora, V. 2009 Turbulence structure of open channel flows over permeable and impermeable beds: A comparative study. Phys. Fluids 21, 125109.

Masaló, I., Guadayol, Ò., Peters, F. \& Oca, J. 2008 Analysis of sedimentation and resuspension processes of aquaculture biosolids using an oscillating grid. Aquacultural Engineering 38, 135-144.

McCorquodale, M. W. \& Munro, R. J. 2017 Experimental study of oscillating-grid turbulence interacting with a solid boundary. J. Fluid Mech. 813, 768-798.

McCorquodale, M. W. \& Munro, R. J. 2018a Analysis of intercomponent energy transfer in the interaction of oscillating-grid turbulence with an impermeable boundary. Phys. Fluids 30, 015105 .

McCorquodale, M. W. \& Munro, R. J. $2018 b$ A method for reducing mean flow in oscillating-grid turbulence. Exp. Fluids 59, 182:1-16.

McDougall, T. J. 1979 Measurements of turbulence in a zero-mean-shear mixed layer. J. Fluid Mech. 94(3), 409-431.

McKenna, S. P. \& McGillis, W. R. 2004 Observations of flow repeatability and secondary circulation in an oscillating grid-stirred tank. Phys. Fluids 16(9), 3499-3502.

Mujal-Colilles, A., Dalziel, S. B. \& Bateman, A. 2015 Vortex rings impinging on permeable boundaries. Phys. Fluids 27, 015106.

Mullens, S., Luyten, J. \& Zeschky, J. 2006 Characterization of Structure and Morphology, pp. 225-266. John Wiley and Sons, Ltd.

Munro, R. J. 2012 The interaction of a vortex ring with a sloped sediment layer: Critical criteria for incipient grain motion. Phys. Fluids 24, 026604.

Munro, R. J., Bethke, N. \& Dalziel, S. B. 2009 Sediment resuspension and erosion by vortex rings. Phys. Fluids 21, 046601.

Musta, M. N. \& Krueger, P. S. 2015 Interaction of steady jets with an array of permeable screens. Exp Fluids 56, 61.

Nield, D. A. \& Bejan, A. 2013 Convection in Porous Media. Springer, New York, NY.

Nokes, R. I. 1988 On the entrainment rate across a density interface. J. Fluid Mech. 188, $185-204$.

Orlandi, P. \& Verzicco, R. 1993 Vortex rings impining on walls: axisymmetric and threedimensional simulations. J. Fluid Mech. 256, 615-646.

Orlins, J. J. \& Gulliver, J. S. 2003 Turbulence quantification and sediment resuspension in an oscillating grid chamber. Exp. Fluids 34, 662-677. 
Perot, B. \& Moin, P. 1995 Shear free turbulent boundary layers, Part 1, physical insights into near-wall turbulence. J. Fluid Mech. 295, 199-227.

Pokrajac, D. \& MANEs, C. 2009 Velocity measurements of a free-surface turbulent flow penetrating a porous medium composed of uniform-size spheres. Transp Porous Med 78, $367-383$.

Rosti, M. E., Brandt, L. \& Pinelli, A. 2018 Turbulent channel flow over an anisotropic porous wall - drag increase and reduction. J. Fluid Mech. 842, 381-394.

Rosti, M. E., Cortelezzi, L. \& Quadrio, M. 2015 Direct numerical simulation of turbulent channel flow over porous walls. J. Fluid Mech. 784, 396-442.

Sharma, A. \& García-Mayoral, R. 2020 Turbulent flows over dense filament canopies. J. Fluid Mech. 888, A2.

Suga, K., Matsumura, Y., Ashitaka, Y., Tominaga, S. \& Kaneda, M. 2010 Effects of wall permeability on turbulence. Int. J. Heat and Fluid Flow 31, 974-984.

Suga, K., Mori, M. \& Kaneda, M. 2011 Vortex structure of turbulence over permeable walls. Int. J. Heat and Fluid Flow 32, 586-595.

Suga, K., NAkAgawa, Y. \& Kaneda, M. 2017 Spanwise turbulence structure over permeable walls. J. Fluid Mech. 822, 186-201.

Szycher, M. 2012 Szycher's handbook of polyurethanes, 2nd edn. Boca Raton, FL : CRC Press.

Thomas, N. H. \& Hancock, P. E. 1977 Grid turbulence near a moving wall. J. Fluid Mech. 82(3), 481-496.

Thompson, S. M. \& Turner, J. S. 1975 Mixing across an interface due to turbulence generated by an oscillating grid. J. Fluid Mech. 67(2), 349-368.

Valsaraj, K.T., Ravikrishna, R., Orlins, J.J., Smith, J.S., Gulliver, J.S., Reible, D.D. \& Thibodeaux, L.J. 1997 Sediment-to-air mass transfer of semi-volatile contaminants due to sediment resuspension in water. Adv. Environ. Res. 1, 145-156.

Voermans, J. J., Ghisalberti, M. \& Ivey, G. N. 2017 The variation of flow and turbulence across the sediment-water interface. J. Fluid Mech. 824, 413-437.

Wagner, C. \& Friedrich, R. 1998 On the turbulence structure in solid and permeable pipes. Int. J. Heat and Fluid Flow 19, 459-469.

Wagner, C. \& Friedrich, R. 2000 Dns of turbulent flow along passively permeable walls. Int. J. Heat and Fluid Flow 21, 489-498.

Walker, D. T., Leighton, R. I. \& Garza-Rios, L. O. 1996 Shear-free turbulence near a flat free surface. J. Fluid Mech. 320, 19-51.

Walker, J. D. A., Smith, C. R., Cerra, A. W. \& Doligalski, T. L. 1987 The impact of a vortex ring on a wall. J. Fluid Mech. 181, 99-140.

Webb, S \& Castro, I. P. 2006 Axisymmetric jets impinging on porous walls. Exp Fluids 40, 951-961.

Whitaker, S 1999 The Method of Volume Averaging. Springer Netherlands.

Yokojima, S. 2011 Effect of wall permeability on wall-bounded turbulent flows. J. Phys. Soc. Jpn. 80, 033401:1-4.

Zhu, W., van Hout, R. \& Katz, J. 2007 PIV measurements in the atmospheric boundary layer within and above a mature corn canopy. Part II: Quadrant-hole analysis. J. Atom. Sci. 64, 2825-2838. 